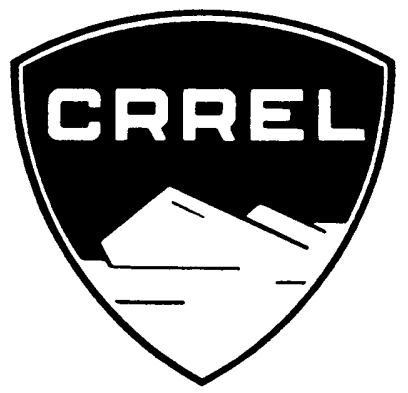

Research Report 87

PLANE PLASTIC DEFORMATION OF SOILS

by

Shunsuke Takagi

JULY 1966

U.S. ARMY MATERIEL COMMAND

COLD REGIONS RESEARCH \& ENGINEERING LABORATORY HANOVER, NEW HAMPSHIRE

DA Task IV014501B 52 A 02

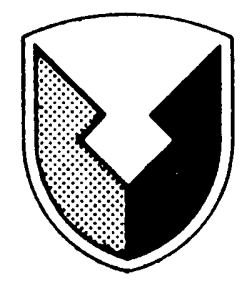

Distribution of this document is unlimited 


\section{PREF ACE}

This report was prepared by Dr. Shunsuke Takagi, Physical Sciences Group, Research Division (J. A. Bender, Chief), U. S. Army" Cold Regions Research and Engineering Laboratory.

USA CRREL is an Army Materiel Command laboratory. 
Page

17, para, $2, \ell, 8,9(2 \widetilde{\sigma} \sin \rho / \gamma)^{\frac{1}{2}}$

should read: $[2 \widetilde{\sigma}(\sin \rho) / \gamma)]^{\frac{1}{2}}$

41, k Darcy constant

should read: Darcy's constant

$41, \delta . \quad 1 / 2<B C D$

should read; $1 / 2$ of the angle BCD 


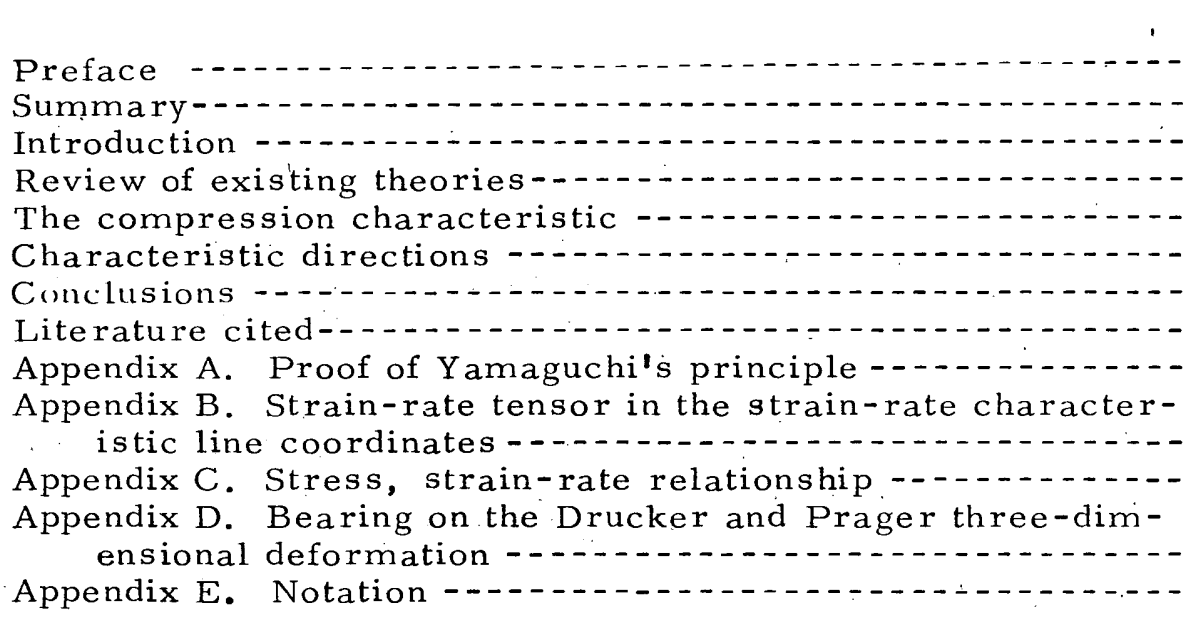

\section{ILLUSTRATIONS}

Figure

1. Sign of tangential stress $\ldots \ldots \ldots$

2. Sign of tangential stress on the Mohr circle

3. Stress characteristic directions--.-.-.-...-.-.

4. Fundamentals of the Geniev deformation --.-.---

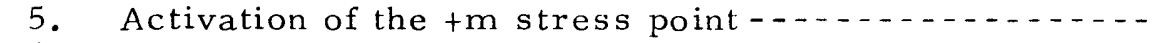

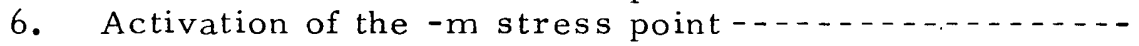

7. Vector field determined by the strain-rate character-

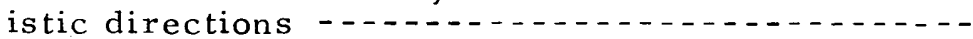

8. The Rankine state under horizontal ground surface---

9. Stress strain-rate relationship in the one-dimensional secondary consolidation ................... 


\section{SUMMARY}

A consistent theory of plane plastic deformation of soil is formulated by assuming soil as an ideal material that has constant cohesion and friction angle. Such an ideal soil is an extension of the ideal metal that has, in the terminology of soil mechanics, cohesion only.

After a review of the existing theories from which the present theory has emerged, the mathematical expression referred to as the "compression characteristic" is developed. Then the system of differential equations is shown by the theory of characteristic lines.

Many mathematical and physical problems remain to be solved before the perfect explanation of the plasticity of ideal soil will be attained. 


\title{
PLANE PLASTIC DEFORMATION OF SOILS
}

by

\author{
Shunsuke Takagi
}

\section{INTRODUCTION}

The mathematical study of the plasticity of an ideal soil having constant cohesion and friction angle, which, for convenience, will be called c- $\phi$ material, has not yet been extensively developed. The objective herein is to present a consistent mathematical basis for the plastic deformation of $\mathrm{c}-\phi$ material with motion limited to in-plane motion.

The theory is developed using only the mathematical requirements that are mandatory to make the strain-rate tensor consistent with the stress tensor. Introduction of these mathematical requirements permits the assignment of suitable characteristics to $c-\phi$ material. These characteristics are: (1) Although the directions of principal stress and principal strain rate are coincident for a plastic metal (Saint-Venant's postulation [Hill, 1956, p. 38]), this is not necessarily so for a c- $\phi$ material; (2) a c- $\phi$ material, depending on its mode of deformation; has either compressibility or dilatancy, which causes change of density; (3) the relationship between stress and strain rate is not determined a priori but in accordance with boundary and initial conditions; (4) it is expected that the three-dimensional yield criterion of $c-\phi$ material may be the form suggested by D. C. Drucker and W. Prager (1952); and (5) it is also expected that limitation of the mode of motion will give various forms of yield criterion.

The differential equations formulated on these bases are confirmed to be consistent by the theory of characteristic lines. They include nonlinear terms caused by local acceleration that, when treated with the theory of characteristic lines, demonstrate that a $c-\phi$ material assumes the motion of a liquid when, under realization of slip lines, a certain velocity is surpassed. This phenomenon is evidenced by the transfer of two of the three characteristic lines from the real to the imaginary. Moreover, these equations include nonsteady terms. Therefore, when adequately developed, they have sufficient generality to become effective eventually in analyzing any type of plane plastic motion of $c-\phi$ material. Two simple examples - vibration under the Rankine state and one-dimensional secondary consolidation - are treated in the text.

Limitations for the practical application of the present theory are: (1) There is no concise experimental method that will provide numerical values of both cohesion and friction angle because the three-dimensional theory remains so obscure that the effect of boundary and initial conditions on plastic behavior during customary tests on soils is uncertain: (2) the deformation of $c-\phi$ material that precedes the plastic deformation, corresponding to elastic deformation in metal, is not combined with the plastic deformation (therefore, we do not know exactly what actual deformation corresponds to the deformation considered herein); and (3) the mathematical proof for existence and uniqueness of the solution is not yet known. These limitations must be removed in the future by enlarging the properties of $c-\phi$ material to make it an idealization of the actual soil.

The letter symbols adopted for use in this paper are defined where they first appear and are listed alphabetically in Appendix E. 


\section{REVIEW OF THE EXISTING THEORIES}

After scrutinizing two apparently irreconcilable approaches for formulating the plasticity of $\mathrm{c}-\phi$ material, the author was able to form a general theory of plane deformation of $c-\phi$ material. One successful method was the extended use (Takagi, 1958-60) of the concept of the pole on the Mohr circle (Terzaghi, 1951). If the stress point of a plane through a given point in the ground is known on the Mohr circle, another intersection with the Mohr circle of the line drawn through the stress, point parallel to the plane is a fixed point called the pole. Conversely, the line drawn from the pole parallel to a plane that passes through the given point in the ground, when it intersects the Mohr circle again, gives the stress point of that plane. However, because the use of this concept largely depends on the location of the stress point, the sign convention for tangential stress must be clearly defined.

If major and minor principal stresses, $\sigma_{I}$ and $\sigma_{I I}$, respectively, are located as shown in Figure 1 , tangential stresses $\tau$ on slopes PQ are directed as shown; slopes $\mathrm{PQ}$ in the left and right.figures are rotated by $\theta$ counterclockwise and clockwise, respectively, from the plane PA of major principal stress.

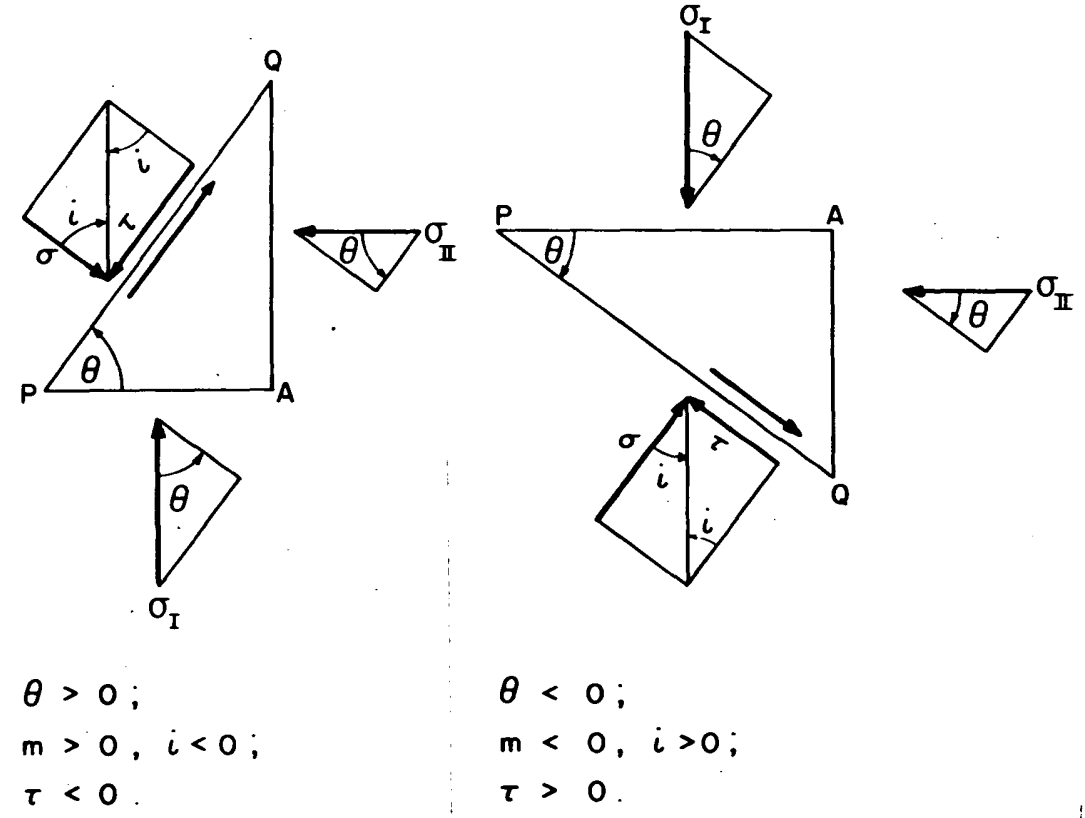

Figure 1. Sign of tangential stress.

In order to express the direction of $\tau$, Terzaghi (1951) uses the angle of inclination, $i$, of compound stress, which, however, is seldom utilized in the application. The better choice is to use the rotational direction of the fictitious moment that is formed by a pair of tangential stresses, $\tau$, located on opposite sides of the same slope because irection is easily determined as a result of agreement with the rotational direction of $\theta$. Moreover, this choice is helpful in visualizing the direction of motion on the slope under consideration because $\tau$ on the slope may be considered as the component of motion that brings $\tau$ into action. The fictitious rotational direction was termed sign $\mathrm{m}$ by the author (Takagi, 1958-60) in order to express the sign of tangential stress. Sign $\underline{m}$ is positive when $\theta$ is positive. 
The application of this concept on the Mohr circle will now be described. In $F$ igure 2 the right-handed and left-handed coordinate systems are shown; $x$ and $y$ are the coordinate axes of the physical plane. The angle $\theta$ in the stress plane is directed in the same manner as $\theta$ in the physical plane. Therefore, $\theta$ is positive in both of the stress planes, and accordingly sign $m$ of $\tau$ at $Q$ in both figures is positive by definition. Angle QOC in Figure 2,

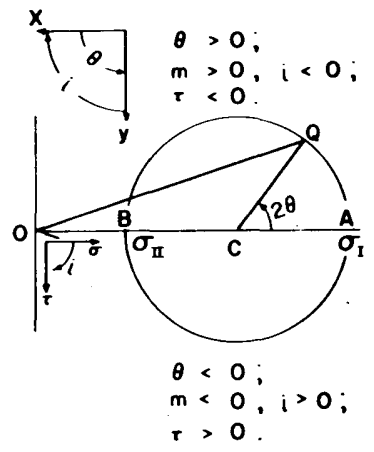

RIGHT - HAND COORDINATE

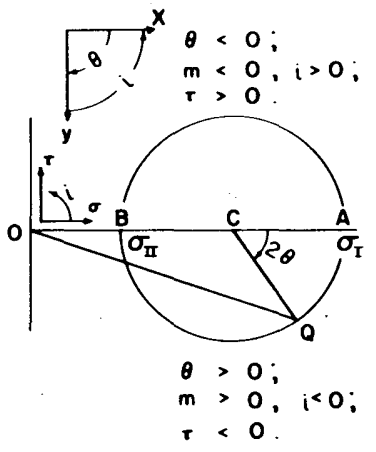

LEFT - HAND COORDINATE

Figure 2. Sign of tangential stress on the Mohr circle.

however, is apparently opposite in direction to $\mathrm{i}$ in Figure 1 , although both should be the same. This discrepancy can be resolved only by directing the $\tau$ axis as ahown in Figure 2 . Then the $\sigma, \tau$ coordinate system obtains the opposite sense from the $x, y$ coordinate system. The following equation is obtained in this manner:

$$
\tau=-(\sigma \sin 2 \theta
$$

where (-) is the radius of the Mohr circle. The relations established so far are shown collectively in Figure 2 .

Once the pole is located on the Mohr circle, the directions of the slip lines, which for consistency will be called stress characteristic directions, are easily determined by the geometric method (Figure 3 ).

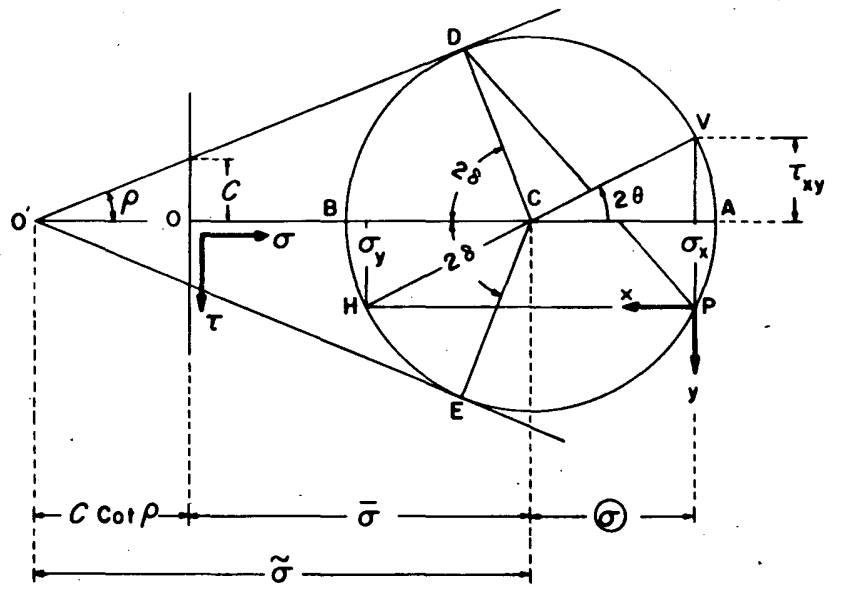

Figure 3. Stress characteristic directions. 
The pole will be located by assuming that the location of the stress point $V$ of the vertical plane has the central angle $2 \theta$ measured from the point $A$ of major principal stress. The next step is to introduce $\delta$, defined by

$$
2 \delta=\frac{\pi}{2}-\rho
$$

where $\rho$ is the frictional angle. Then, from the geometry in Figure 3,

$$
<\mathrm{DPH}=-\theta-\delta
$$

and

$$
<\mathrm{EPH}=-\theta+\delta
$$

where $D$ and $E$ are the $+m$ and $-m$ stress points, respectively, on the limiting lines, and $\mathrm{H}$ is the stress point of the horizontal plane. Stress characteristic directions $\mathrm{PD}$ and $\mathrm{PE}$ are therefore given as

$$
d y=-\tan (\theta \pm \delta) d x
$$

where the upper and lower of the double signs indicate the $+m$ and $-m d i-$ rections, respectively. This convention of double signs will be used throughout this paper unless otherwise stated.

Equation 4 has been derived using the right-handed coordinate system with the $y$ axis directed vertically downward. It is also valid (if the convention shown in Figure 2 is followed) not only for the right-handed coordinate system with the $y$ axis directed vertically upward, but also for the lefthanded coordinate system with the y direction either vertically downward or upward. It should be noted that the $\sigma$ axis does not have to be horizontal in order to apply the geometry of the Mohr circle; however, slight complications must be disposed of if the $\sigma$ axis is not horizontal.

When the sense of the $\sigma, \tau$ coordinate system is observed, the theory of characteristic lines provides the same stress characteristic direction as given by the geometric method. This may be verified by using Sokolovski's method (Sokolovski, 1960), or by using the method presented subsequently herein. The negative sign in front of $\tan (\theta \pm \delta)$ does not appear in Sokolovski's equations because of the different definition for $\theta$ (Sokolovski, 1960, eq 1.15 and 1.16). If it is desired to remove the negative sign, $\theta$ should be defined as one-half the central angle of the pole, or as the angle from the horizontal to the line of action of $\sigma_{I}$. This choice, however, is not convenientfor manipulating the geometry of the Mohr circle.

The basic assumption for plastic deformation adopted in the general theory of plasticity is that the strain rate, or, if not referring to time, the strain increment, is determined by the stress, whereas in elastic deformation the strain itself is related to stress (Yoshimura, 1959a, b; Green and Zerna, 1954).

In the case of a metal obeying the Mises criterion of yielding (in which $\mathrm{J}_{2}$ is equal to a constant) the relation between a component of strain rate $\dot{\epsilon}_{i j}$ and components of stress $\sigma_{i j}$ is found by differentiating the yield function $\mathrm{J}_{2}$ in respect to $\sigma_{i j}$, that is,

$$
\dot{\epsilon}_{i j}=f \frac{\partial J_{2}}{\partial \sigma_{i j}}
$$


where $J_{2}$ is the second invariant of the deviatoric stress tensor,

$$
J_{2}=\frac{1}{6}\left[\left(\sigma_{1}-\sigma_{2}\right)^{2}+\left(\sigma_{2}-\sigma_{3}\right)^{2}+\left(\sigma_{3}-\sigma_{1}\right)^{2}\right]
$$

where $\sigma_{1}, \sigma_{2}$, and $\sigma_{3}$ are the principal stresses, arranged in an arbitrary order; $\mathrm{f}^{1}$ is a positive constant; and the dot on $\dot{\epsilon}_{i j}$ signifies differentiation with respect to time. When regarded as a function of the components of stress, $J_{2}$ is called plastic potential. It must be noted, however, that in deriving eq 5 Saint-Venant's postulate (Hill, 1956) is presumed.

Drucker and Prager (1952) extended the concept of plastic potential to the three-dimensional deformation of $c-\phi$ material, showing that

$$
\mathrm{J}_{2}^{1 / 2}-\lambda \mathrm{J}_{1}=\mathrm{k}
$$

is a proper generalization of the Mises criterion of yielding (Drucker, 1953), and

$$
\psi_{\mathrm{d}}=\mathrm{J}_{2}{ }^{1 / 2}-\lambda \mathrm{J}_{1}
$$

is the plastic potential for this case, in which $\lambda$ and $k$ are positive constants and $\mathrm{J}_{1}$ is the first invariant,

$$
J_{1}=\sigma_{1}+\sigma_{2}+\sigma_{3}
$$

It should be noted that a positive sign is used to indicate compressive stress herein. In the case of plane strain, the yield criterion (eq 7) is reduced to the Mohr criterion of yielding. It is noted that Saint-Venant's postulate is still maintained.

Drucker and Prager's theory was advanced further by Drucker (1953) and Shield (1953) for the case of plane deformation, but could not be widely accepted by researchers in soil mechanics. The reason may be that it was not clear whether Drucker and Prager's derivation from the threeddimensional extension of the yield criterion was adequate for the plane deformation of $c-\phi$ material (Hansen 1953). Yamaguchi (1959) almost succeeded in indicating that, if Saint-Venant's postulate is presumed, the Drucker and Prager extension of the Mohr criterion of yielding is the only possible yield criterion for the three-dimensional deformation of $c-\phi$ material (see Appendixes $C$ and $D$ ).

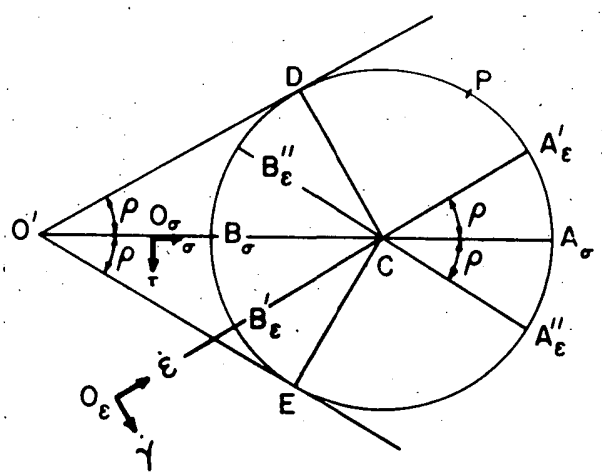

Figure 4. Fundamentals of the Ceniev deformation.
Geniev (1958) developed another theory of plane deformation. Geniev's fundamental concept is most easily explained geometrically, as shown in Figure 4. If both the scale and the position of the $\dot{\epsilon}, \dot{r}$ axes are adequately selected, in which $r$ is shear strain rate, the Mohr circle of strain rate may be superimposed on the Mohr circle of stress with the poles of both circles fixed at the same point (for example, P) so that the geometric interpretation is feasible. The stress point at which the plastic defor: mation is operative is,' as is well recognized, either $D$ or $E$ where the limiting, lines touch the Mohr circle, whereas the strain-rate 
point at which the plastic deformation is operative, called the operative strain-rate point, is not known. However, Geniev arbitrarily assumed (1) that the operative strain-rate point would be the maximum shear strainrate point, and (2) that the operative strain-rate point would fall on the stress point of the same sign $m$ on one of the limiting lines. Then, either $P D$ or $P E$ is the common characteristic direction. The $\dot{\epsilon}$ axis appears on either $A_{\epsilon}^{\prime} B_{\epsilon}^{\prime}$ or $A_{\epsilon}^{\prime \prime} B_{\epsilon}^{\prime \prime}$, which are rotated by $\rho$ counterclockwise and clockwise, respectively, from the $\sigma$ axis. Thus, as was suggested by de Jong (1958), Geniev deviated from Saint-Venant's postulate adopted for metallurgy.

Although Geniev's first assumption is arbitrary, the second assumption is mandatory. If two distinct characteristic directions exist, one from stress and one from strain rate, it is not possible to satisfy conditions on boundary lines, or to connect two discontinuous solutions across a line. Coincidence of both strain-rate characteristic directions with the stress characteristic directions of the same sign $\underline{m}$, as in the Drucker and Prager deformation, is not necessary.

In order to formulate the plastic deformation, Geniev's second as sumption is adopted as the first basic assumption herein. The arbitrary location of the operative strain-rate point on the strain-rate Mohr circle is replaced by the following principle, which Yamaguchi (1959) inductively adopted in deriving the Drucker and Prager plane deformation, although not in a perfect form, from the Mohr criterion of yielding on the basis of Saint-Venant's postulate. The transformation of a set of first-order partial differential equations to a coordinate system in which the coordinates are the characteristic lines of the given equations is simplification of the set of equations into a set in which each equation contains directional differentials in only one coordinate. The set of transformed equations may be expressed as a set of total differential equations, each of which is valid along a characteristic direction. This is a mathematical fact (Appendix A), and is adopted herein as the second basic assumption.

\section{THE COMPRESSION CHARACTERISTIC}

Let us suppose that the strain-rate Mohr circle and the stress Mohr circle are superimposed with their poles at the same point $P$ (Fig. 5; 6). Then, according to the first basic assumption, the operative strain + rate point must appear on either $D$ in Figure 5 or $E$ in Figure 6 , which (since the $x, y$ coordinate system is assumed here to be the right-handed coordinate system) is either the $+m$ or the $-m$ stress point, respectively. The stress point on which the operative strain-rate point occurs will be termed activated.

The location of the inoperative strain-rate point $\left(\mathrm{E}_{\epsilon}\right.$ in Figure 5 or $\mathrm{D}_{\epsilon}$ in Figure 6), however, is not known a priori. The central angle of $\mathrm{E}_{\epsilon}$ or $D_{\epsilon}$ is expressed by assuming that it is larger by $2 \phi$ than that of the $-\mathrm{m}^{\epsilon}$ or the $+m$ stress point on the limiting lines $\left(E_{\sigma}\right.$ in Figure 5 or $D_{\sigma}$ in Figure 6 ). The central angle of the point $A_{\epsilon}$ of major principal strain rate is expressed by assuming that it is larger by $\epsilon^{2}$ a than that of the point $A_{\sigma}$ of major principal stress. The immediate objective of the following analys is is to determine what relationship must exist between $\phi$ and $a$ in order that the strainrate characteristics conform to the stress characteristics. 


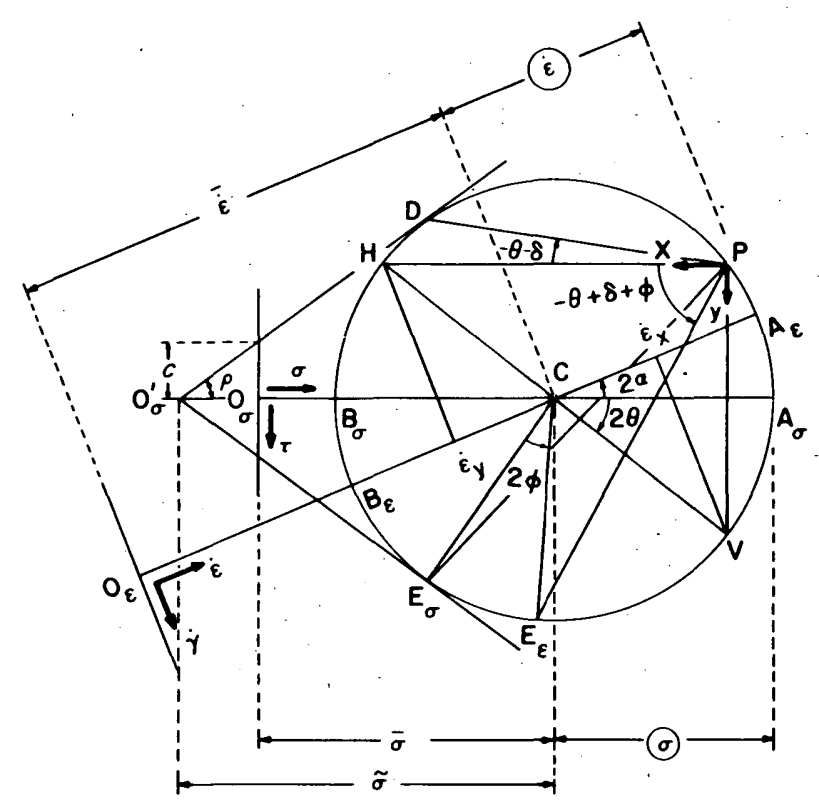

Figure 5. Activation of the $+m$ stress point.

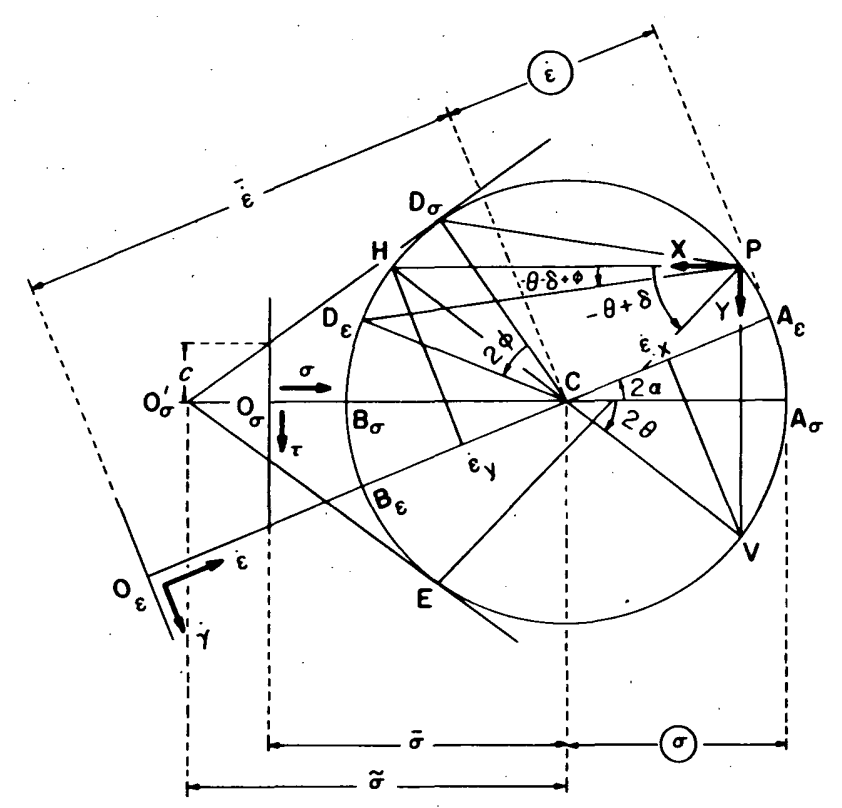

Figure 6. Activation of the $-m$ stress point.

From the geometry of Figures 5 and 6,

$$
\begin{aligned}
& \dot{\epsilon}_{x}=\overline{\dot{\epsilon}}+(\dot{\epsilon} \cos (2 \theta-2 a) \\
& \dot{\epsilon}_{y}=\overline{\dot{\epsilon}}-(\dot{\xi} \cos (2 \theta-2 a)
\end{aligned}
$$

and

$$
\dot{r}_{\mathrm{xy}}=-(\dot{\epsilon} \sin (2 \theta-2 a)
$$

where () and $\overline{\dot{\epsilon}}$ are the radius and the $\dot{\epsilon}$ coordinate of the center of the strain-rate Mohr circle, and the negative sign in the right-hand side of eq 12 is used in accordance with the sign convention for $\boldsymbol{\tau}_{\mathrm{xy}}$.

Components of strain rate are assumed to be positive when they are compressive. Components of velocity are positive in the positive $\underline{x}$ or $\mathbf{y}$ directions, and are connected by the relations,

$$
\begin{aligned}
& \dot{\boldsymbol{\epsilon}}_{\mathrm{x}}=-\frac{\partial \mathrm{v}_{\mathrm{x}}}{\partial \mathrm{x}} \\
& \dot{\boldsymbol{\epsilon}}_{\mathrm{y}}=-\frac{\partial \mathrm{v}_{\mathrm{y}}}{\partial \mathrm{y}}
\end{aligned}
$$

and

$$
\dot{r}_{x y}=-\frac{1}{2}\left(\frac{\partial v_{x}}{\partial y}+\frac{\partial v_{y}}{\partial x}\right) .
$$


Eliminating $\overline{\dot{\epsilon}}$ and $(\dot{\epsilon}$ in eq 10,11 , and 12 and using eq 13, 14, and 15, yields the following:

$$
\left(\frac{\partial v_{x}}{\partial x}-\frac{\partial v y}{\partial y}\right) \quad \sin (2 \theta-2 a)+\left(\frac{\partial v x}{\partial y}-\frac{\partial v y}{\partial x}\right) \cos (2 \theta-2 a)=0 .
$$

Equation 16 is the only equation that connects components of velocity and, by Yamaguchi's principle (p. 6), it must become total differential equations when expressed in terms of strain-rate characteristic directions.

For a consistent analysis, reference is made to the vector field determined by the strain-rate characteristic directions, schematically represented in Figure 7 , in which $\overrightarrow{s_{0}}$ and $\overrightarrow{s_{1}}$ are the operative and the inoperative directions, respectively. The angles that these strain-rate charac-

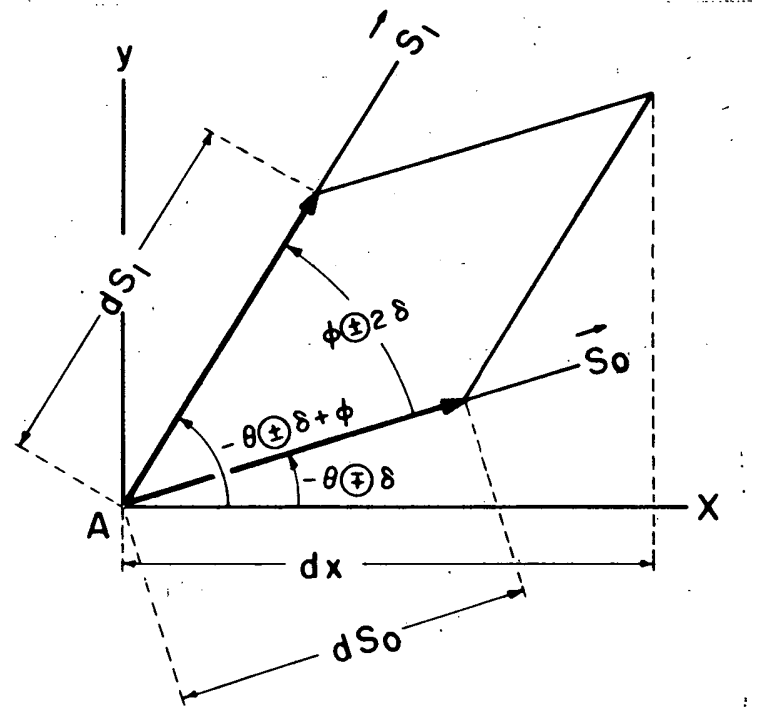

Figure 7. Vector field determined by the strain-rate characteristic directions. teristics make with the $x$ direction are easily determined from the geometry in Figure 5 or 6 , in which the operative direction is $P D$, or $P E$ and the inoperative direction is $\mathrm{PE}$ or $\mathrm{PD}_{\epsilon}$, respectively. The $\mathrm{re}^{-}$ sult is summarized as $-\theta(\delta$ for the operative and $-\theta \biguplus \delta+\phi$ for the inoperative, where the upper or the lower of the double signs in the circle corresponds to the activation of the $+m$ or the -m stress characteristic direction, respectively, and the circle surrounding the double signs signifies that only one of the double signs is actually used.

The relationship between the vectors $(\mathrm{dx}, \mathrm{dy})$ and $\left(\mathrm{ds}, \mathrm{ds}_{1}\right)$ gives the relationship between the directional differentiations,

$$
\begin{aligned}
& \sin (\phi \Theta 2 \delta) \frac{\partial}{\partial x}=-\sin (\theta \bigoplus \delta-\phi) \frac{\partial}{\partial s_{0}}+\sin (\theta \Theta \delta) \frac{\partial}{\partial s_{1}} \\
& \sin (\phi \Theta 2 \delta) \frac{\partial}{\partial y}=-\cos (\theta \bigoplus \delta-\phi) \frac{\partial}{\partial s_{0}}+\cos (\theta \bigoplus \delta) \frac{\partial}{\partial s_{1}}
\end{aligned}
$$

It should be noted that the differentiation with respect to $s_{0}$ and $s_{1}$ does not indicate that the quantity to be differentiated is expressible as a function of $s_{0}$ and $s_{1}$. This is evident from the fact that the order of differentiation in $\partial^{2} / \partial s{ }_{0} \partial s$ usually cannot be reversed. The differentiation with respect to $s$ and $s$ shall be understood to signify the rate of change of quantity in the respective directions.

Substitution of relationships 17 and 18 into eq 16 yields

$$
-\left[\frac{\partial v_{x}}{\partial s_{0}} \cos (\theta \Theta \delta-2 a+\phi)-\frac{\partial v_{y}}{\partial s_{0}} \sin (\theta \Theta \delta-2 a+\phi)\right]+
$$




$$
+\left[\frac{\partial v_{x}}{\partial s_{1}} \cos (\theta \subsetneq \delta-2 a)-\frac{\partial v_{y}}{\partial s_{l}} \sin (\theta \odot \delta-2 a)\right]=0
$$

Equation 19. is not a total differential equation, and the sums of the terms within each set of brackets must be zero in order to yield two total differential equations. The two total differential equations thus obtained may be written in the following forms:

$$
d v_{x} \cos (\theta \Theta \delta-2 a+\phi)-d v_{y} \sin (\theta \bigoplus \delta-2 a+\phi)=0
$$

along the operative strain-rate characteristic direction, where

$$
\frac{d \dot{x}}{\cos (\theta \oplus \delta)}=\frac{d y}{-\sin (\theta \Theta \delta)}=\mathrm{ds}_{0}
$$

and

$$
d v_{x} \cos (\theta \bigoplus \delta-2 a)-d v_{y} \sin (\theta \bigoplus \delta-2 a)=0
$$

along the inoperative strain-rate characteristic direction, where

$$
\frac{d x}{\cos (\theta \mp \delta-\phi)}=\frac{d y}{-\sin (\theta \mp \delta-\phi)}=d s_{1} \text {. }
$$

Thus, two conditions have been obtained beginning from one relationship (eq 16) and the existence of another relationship should be expected. To write this other relationship, substitute

$$
\begin{aligned}
& d v_{x}=\frac{\partial v_{x}}{\partial x} d x+\frac{\partial v_{x}}{\partial y} d y \\
& d v_{y}=\frac{\partial v_{y}}{\partial x} d x+\frac{\partial v_{y}}{\partial y} d y
\end{aligned}
$$

into eq 20 and 22 and use eq 21 and 23 to eliminate $d v / d x$. Solving the two equations thus obtained simultaneously for $\partial v_{x} / \partial x$ and $\partial v_{y} / \partial_{y}$, and adding the resultant two equations, the following is obtained:

$$
\begin{aligned}
& \left(\frac{\partial v_{x}}{\partial x}+\frac{\partial v_{y}}{\partial y}\right) \cos (2 a-\phi) \sin (2 \theta-2 a) \\
& =\cos (\phi \oplus 2 \delta)\left(\frac{\partial v_{x}}{\partial y}+\frac{\partial v_{y}}{\partial x}\right) \\
& +\sin (2 \theta-2 a) \sin (\phi-2 a)\left(\frac{\partial v_{y}}{\partial x}-\frac{\partial v}{\partial y}\right) .
\end{aligned}
$$

This relationship must be an invariant of strain-rate tensor, and the second term in the right-hand-member must vanish. . This requirement is fulfilled by setting

$$
\phi=2 a .
$$


Then eq 25 changes to

$$
\overline{\dot{\epsilon}}=-\Theta \cos (2 a \oplus 2 \delta)
$$

using the expressions of $\dot{\gamma}_{x y}$ in eq 12 and 15 . This relationship will provide a systematic treatment for the plasticity of $c-\phi$ material and henceforth will be referred to as the compression characteristic.

Because $\overline{\dot{\epsilon}}$ indicates the rate of volume decrease and $(\dot{\xi}$ is positive, either dilatancy or compression must accompany the plastic deformation of $c-\phi$ material in accordance with $\cos (2 a \notin 2 \delta)>$ or $\langle 0$, respectively. For the particular case of $2 a=\oplus \rho$, a change in volume does not occur; this is the deformation described by Geniev (1958). In the case of the Drucker and Prager plane deformation, in which $2 a=0$, dilatancy always occurs, as Drucker and Prager have stated.

Equation 26 stipulates the following geometric interpretation; the inoperative strain-rate point is symmetric with the operative strain-rate point in regard to the $\dot{\epsilon}$ axis on the strain-rate Mohr circle.

Equation 26 simplifies eq 20 and 22 to the same form,

$$
d v_{x} d x+d v_{y} d y=0
$$

in which eq 21 is valid along the operative strain-rate characteristic direction, and

$$
\frac{d x}{\cos (\theta \mp \delta-2 a)}=\frac{d y}{-\sin (\theta \circledast \delta-2 a)}=\mathrm{ds}_{1}
$$

is valid along the inoperative strain-rate characteristic direction. Reduction to the simple form of eq 28 results in the following interpretations: (1) The increment of velocity, of which the components are $d v_{x}$ and $d v_{y}$, along each of the strain-rate characteristic directions is normal to the respective strain-rate characteristic direction, of which the components are $\mathrm{dx}$ and dy; and (2) the unit compression rates $\dot{\theta}_{0}$ and $\dot{\epsilon}_{1}$ in the operative and the inoperative directions, respectively, are both zero, as may be verified by substitution of eq $24 \mathrm{a}$ and $24 \mathrm{~b}$ into eq 28 . However, the shear strain rate $\dot{\gamma}_{01}$ in the coordinates of the strain rate characteristic line is not zero (see Appendix B) and therefore $\overline{\dot{\epsilon}}$ is not zero.

The following theorem can be presented in a manner similar to the derivation of eq 25 . If eq 28 is assumed to be valid in both the operative and the inoperative directions, both eq 16 and 27 must be valid. Therefore, eq 28 is equivalent to eq 16 and 27.

\section{CHARACTERISTIC DIRECTIONS}

Fundamental equations governing the plastic deformation of $\mathrm{c}-\phi$ material are as follows:

Writing $\overline{O^{\prime} C}=\tilde{\sigma}$ in Figure 3 , and from the geometry of the stress Mohr circle, the following is obtained:

$$
\begin{aligned}
& \sigma_{x}=\tilde{\sigma}(1+\sin \rho \cos 2 \theta)-c \cot \rho \\
& \sigma_{y}=\sigma_{(1-\sin \rho \cos 2 \theta)-c \cot \rho}
\end{aligned}
$$


and

$$
\tau_{\mathrm{xy}}=-\tilde{\sigma} \sin \rho \sin 2 \theta
$$

where c denotes cohesion. The stress, strain-rate relationship is obtained $\bar{b}$ y eliminating $\widetilde{\sigma}, \theta$, and $\dot{\epsilon}$ in eq $30,31,32,10,11,12$, and 27 (see Appendix C). It is noted that $a$ is generally a function of location and time, and that the variability of a leads to particular attributes of $c-\phi$ material.

The equations of motion are

$$
\begin{aligned}
& \frac{1}{r}\left(\frac{\partial \sigma_{x}}{\partial x}+\frac{\partial \tau}{\partial y}\right)+v_{x} \frac{\partial v_{x}}{\partial x}+v_{y} \frac{\partial v_{x}}{\partial y}+\frac{\partial v_{x}}{\partial t}=x \\
& \frac{1}{r}\left(\frac{\partial \tau}{\partial x}+\frac{\partial \sigma y}{\partial y}\right)+v_{x} \frac{\partial v_{y}}{\partial x}+v_{y} \frac{\partial v_{y}}{\partial y}+\frac{\partial v_{y}}{\partial t}=Y
\end{aligned}
$$

and the equation of continuity is

$$
\frac{\partial \gamma}{\partial t}+\frac{\partial\left(r v_{x}\right)}{\partial x}+\frac{\partial\left(r v_{y}\right)}{\partial y}=0
$$

where (since either dilatancy or compression always occurs) the unit density $\gamma$ is a function of the variables $\underline{x}, \underline{y}$, and $\underline{t}$; and $X$ and $Y$ are the external forces on the unit mass in the $\underline{x}$ and $\underline{y} \bar{d}$ irections, respectively.

The plastic work rate

$$
\dot{W}=\sigma_{x} \dot{\epsilon}_{x}+2 \tau_{x y} \dot{\gamma}_{x y}+\sigma_{y} \dot{\epsilon}_{y}
$$

must remain non-negative during the plastic deformation of $c-\phi$ material if no irreversible changes other than the plastic deformation are concurrent. Upon substitution of eq $30,31,32,10,11,12$ and 27 and the equation,

$$
\sigma=\tilde{\sigma} \sin \rho
$$

$\dot{\mathrm{W}}$ is found to be

$$
\dot{\mathrm{W}}=2 \Theta \cot \rho[\mathrm{c} \cos (2 a \oplus 2 \delta) \oplus \Theta \sin 2 a]
$$

where (O) is the radius of the stress Mohr circle. Therefore, the inequality condition

$$
\text { (E) }[c \cos (2 a \oplus 2 \delta) \oplus \odot \sin 2 a] \geqq 0
$$

must be satisfied. The consistency of this system of equations is shown in the following analysis: Inserting eq 30,31 , and 32 into eq 33 and 34 yields

$$
\begin{gathered}
P_{1} \frac{1}{\gamma} \frac{\partial \tilde{\sigma}}{\partial x}+Q_{1} \frac{1}{r} \frac{\partial \tilde{\sigma}}{\partial y}+R_{1} \frac{1}{r} 2 \tilde{\sigma} \sin \rho \frac{\partial \theta}{\partial x}+S_{1} \frac{1}{r} 2 \tilde{\sigma} \sin \rho \frac{\partial \theta}{\partial y}+v_{x} \frac{\partial v_{x}}{\partial x}+ \\
+v_{y} \frac{\partial v_{x}}{\partial y}=x-\frac{\partial v_{x}}{\partial t}
\end{gathered}
$$


and

$\mathrm{P}_{2} \frac{1}{\gamma} \frac{\partial \tilde{\sigma}}{\partial \mathrm{x}}+\mathrm{Q}_{2} \frac{1}{\gamma} \frac{\partial \tilde{\sigma}}{\partial \mathrm{y}}+\mathrm{R}_{2} \frac{1}{\gamma} 2 \tilde{\sigma} \sin \rho \frac{\partial \theta}{\partial \mathrm{x}}+\mathrm{S}_{2} \frac{1}{\gamma} 2 \tilde{\sigma} \sin \rho \frac{\partial \theta}{\partial \mathrm{y}}+\mathrm{v}_{\mathrm{x}} \frac{\partial \mathrm{v}_{\mathrm{y}}}{\partial \mathrm{x}}+$

$$
+v_{y} \frac{\partial v_{y}}{\partial y}=Y-\frac{\partial v_{y}}{\partial t}
$$

where

$$
\begin{aligned}
& \mathrm{P}_{1}=1+\sin \rho \cos 2 \theta \\
& \mathrm{Q}_{1}=-\sin \rho \sin 2 \theta \\
& \mathrm{R}_{1}=-\sin 2 \theta \\
& \mathrm{S}_{1}=-\cos 2 \theta \\
& \mathrm{P}_{2}=-\sin \rho \sin 2 \theta \\
& \mathrm{Q}_{2}=1-\sin \rho \cos 2 \theta \\
& \mathrm{R}_{2}=-\cos 2 \theta
\end{aligned}
$$

and

$$
\mathrm{S}_{2}=\sin 2 \theta \text {. }
$$

Eliminating $\bar{\epsilon}$ and $(\epsilon)$ in eq 10,11 , and 12 , and using eq 13,14 , and 15 yields

$$
A_{1} \frac{\partial v_{x}}{\partial x}+B_{1} \frac{\partial v_{x}}{\partial y}+C_{1} \frac{\partial v y}{\partial x}+D_{1} \frac{\partial v y}{\partial y}=0
$$

By eliminating () and using the expression for $\dot{r}_{x y}$ in eq 12 , eq 27 changes

$$
A_{2} \frac{\partial v_{x}}{\partial x}+B_{2} \frac{\partial v_{x}}{\partial y}+C_{2} \frac{\partial v_{y}}{\partial x}+D_{2} \frac{\partial v_{y}}{\partial y}=0
$$

where

$$
\begin{aligned}
& A_{1}=\sin (2 \theta-2 a) \\
& B_{1}=\cos (2 \theta-2 a) \\
& C_{1}=\cos (2 \theta-2 a) \\
& D_{1}=-\sin (2 \theta-2 a) \\
& A_{2}=\sin (2 \theta-2 a) \\
& B_{2}=-\cos (2 a \oplus 2 \delta) \\
& C_{2}=-\cos (2 a \oplus 2 \delta)
\end{aligned}
$$


and

$$
D_{2}=\sin (2 \theta-2 a) \text {. }
$$

In addition, the conditions of one-valuedness for the unknowns, $\tilde{\sigma}, \theta, v_{\mathbf{x}}$, $v_{y}$, and $r$ must be satisfied:

$$
\begin{aligned}
\mathrm{d} \tilde{\sigma} & =\frac{\partial \tilde{\sigma}}{\partial \mathrm{x}} \mathrm{dx}+\frac{\partial \tilde{\sigma}}{\partial \mathrm{y}} \mathrm{dy} \\
\mathrm{d} \theta & =\frac{\partial \theta}{\partial \mathrm{x}} \mathrm{dx}+\frac{\partial \theta}{\partial \mathrm{y}} \mathrm{dy} \\
\mathrm{dv}_{\mathrm{x}} & =\frac{\partial \mathrm{v}_{\mathrm{x}}}{\partial \mathrm{x}} \mathrm{dx}+\frac{\partial \mathrm{v}_{\mathrm{x}}}{\partial \mathrm{y}} \mathrm{dy} \\
\mathrm{dv}_{\mathrm{y}} & =\frac{\partial \mathrm{v}_{\mathrm{y}}}{\partial \mathrm{x}} \mathrm{dx}+\frac{\partial v_{\mathrm{y}}}{\partial \mathrm{y}} \mathrm{dy}
\end{aligned}
$$

and

$$
d r=\frac{\partial r}{\partial x} d x+\frac{\partial r}{\partial y} d y
$$

The ten equations in eq $35,40,41,50,51$, and 60 through 64 contain ten local partial derivatives of $\tilde{\sigma}, \theta, v_{x}, v_{y}$, and $\gamma$ with respect to $x$ and $y$. The determinant of coefficients $(\Delta)$ of the ten simultaneous (algebraic) linear equations with the ten local derivatives as unknowns is calculated and found to be equal to

$$
\Delta=\Delta_{\sigma} \Delta_{\epsilon}\left(v_{x} d y-v_{y} d x\right)
$$

where

$$
\Delta_{\sigma}=\left|\begin{array}{cccc}
P_{1} & Q_{1} & R_{1} & S_{1} \\
P_{2} & Q_{2} & R_{2} & S_{2} \\
d x & d y & 0 & 0 \\
0 & 0 & d x & d y
\end{array}\right| \text { and } \Delta_{\epsilon}=\left|\begin{array}{cccc}
A_{1} & B_{1} & C_{1} & D_{1} \\
A_{2} & B_{2} & C_{2} & D_{2} \\
d x & d y & 0 & 0 \\
0 & 0 & d x & d y
\end{array}\right| \text {. }
$$

Characteristic directions are determined by placing $\Delta$ equal to zero. One of the characteristic directions is the stream line, in which $\mathrm{dx} / \mathrm{v}=$ $=\mathrm{dy} / \mathrm{v}_{\mathrm{y}}$ From $\Delta_{\sigma}=0$, the stress-characteristic directions already $\mathrm{x}_{i v e n}$ by eq 4 are determined. From $\Delta_{\epsilon}=0$, the operative and the inoperative strain-rate characteristic directions already given by eq 21 and 29 , respectively, are determined. It is then obvious that eq 28 is also valid for this case in which the change of both stress and density is concurrent with the change of velocity.

For purposes of consistency in the following paragraphs, a transformation of eq 28 is added here by placing

$$
v_{x}=v \cos \omega .
$$


and

$$
v_{y}=v \sin \omega
$$

where $\mathrm{v}$ is the magnitude of compound velocity and $\omega$ denotes the angle measured from the $x$ axis to the direction of velocity. Equation 28 then changes to

$$
\mathrm{dv} / \mathrm{v}=\tan (\theta \Theta \delta+\omega) \mathrm{d} \omega
$$

along the operative strain-rate characteristic direction, expressed by eq 21 , and to

$$
\mathrm{dv} / \mathrm{v}=\tan (\theta \oplus \delta-2 a+\omega) \mathrm{d} \omega
$$

along the inoperative strain-rate characteristic direction, expressed by eq 29.

One of the most interesting subjects in the plastic motion of $c-\phi$ material is the realization of slip lines, in which a particular phenomenon may occur that cannot be seen in the plastic motion described in previous paragraphs. A slip line is a flow line of the material that is realized when the direction of velocity coincides with the activated stress characteristic direction, or, in other words, with the operative strain-rate characteristic direction.

When the slip line is realized,

$$
\omega=-\theta \oplus \delta+\mathrm{n} \pi \text {. }
$$

Then, from eq 67 ,

$$
\mathrm{dv}=0
$$

on the slip line, of which the direction is given by eq 21 . In other words, velocity is constant along the slip line, which is a remarkable feature of $c-\phi$ material. From eq 68 ,

$$
\mathrm{dv} / \mathrm{v}=\tan (2 \mathrm{a} \oplus 2 \delta) \mathrm{d} \theta
$$

in the inoperative strain-rate characteristic direction, given by eq 29. Particularly in the Geniev deformation (in which $2 a=\oplus \rho$ ) the inoperative is the equi- $\theta$ direction, because $d \theta$ must be zero to maintain the right side of eq 71 at a finite value. For the realization $q f$ slip lines, the strainrate characteristic directions and the total differential equations along these directions are obtained in this manner as a particular case of the plastic motion in general.

Characteristic directions for realization of slip lines other than those previously mentioned are obtained by writing

$$
\mathrm{v}_{\mathrm{x}}=\mathrm{v} \cos (\theta \oplus \delta)
$$

and

$$
v_{y}=-v \sin (\theta \oplus \delta)
$$


allowing $\mathrm{v}$ to be either positive or negative. By substituting eq 72 and 73 into eq $3 \overline{3}$ and 34 , the following is obtained:

$$
\mathrm{P}_{1} \frac{\partial \tilde{\sigma}}{\partial \mathrm{x}}+\mathrm{Q}_{1} \frac{\partial \tilde{\sigma}}{\partial \mathrm{y}}+\mathrm{R}_{1} 2 \tilde{\sigma} \sin \rho \frac{\partial \theta}{\partial \mathrm{x}}+\mathrm{S}_{1} 2 \tilde{\sigma} \sin \rho \frac{\partial \theta}{\partial \mathrm{y}}=r\left(\mathrm{x}-\frac{\partial \mathrm{v}_{\mathrm{x}}}{\partial t}+2 \overline{\dot{\varepsilon}} \mathrm{v}_{\mathrm{x}}\right)(74)
$$

and

$$
\mathrm{P}_{2} \frac{\partial \tilde{\sigma}}{\partial \mathrm{x}}+\mathrm{Q}_{2} \frac{\partial \tilde{\sigma}}{\partial \mathrm{y}}+\mathrm{R}_{2} 2 \tilde{\sigma} \sin \rho \frac{\partial \theta}{\partial \mathrm{x}}+\mathrm{S}_{2} 2 \tilde{\sigma} \sin \rho \frac{\partial \theta}{\partial \mathrm{y}}=r\left(\mathrm{Y}-\frac{\partial \mathrm{v}_{\mathrm{y}}}{\partial \mathrm{t}}+2 \overline{\dot{\epsilon}} \mathrm{v}_{\mathrm{y}}\right)
$$

where

$$
\begin{aligned}
& \mathrm{P}_{1}=1+\sin \rho \cos 2 \theta \\
& \mathrm{Q}_{1}=-\sin \rho \sin 2 \theta \\
& \mathrm{R}_{1}=-\sin 2 \theta \\
& \mathrm{S}_{1}=-\cos 2 \theta+\nu^{2} \\
& \mathrm{P}_{2}=-\sin \rho \sin 2 \theta \\
& \mathrm{Q}_{2}=1-\sin \rho \cos 2 \theta \\
& \mathrm{R}_{2}=-\cos 2 \theta-\nu^{2} \\
& \mathrm{~S}_{2}=\sin 2 \theta \\
& \nu^{2}=\frac{\gamma v^{2}}{2 \gamma^{2} \sin \rho}
\end{aligned}
$$

and

$$
\overline{\dot{\epsilon}}=-\frac{1}{2} \frac{\partial v_{x}}{\partial x}+\frac{\partial v y}{\partial y} .
$$

In deriving eq 74 and 75 , the relationships

$$
v_{x} \frac{\partial v_{x}}{\partial x}+v_{y} \frac{\partial v_{x}}{\partial y}+2 \overline{\dot{\epsilon}} v_{x}=v^{2} \frac{\partial \theta}{\partial y}
$$

and

$$
v_{x} \frac{\partial v_{y}}{\partial x}+v_{y} \frac{\partial v_{y}}{\partial y}+2 \overline{\dot{\epsilon}} v_{y}=-v^{2} \frac{\partial \theta}{\partial x}
$$

which are easily verified by the use of eq 72,73 , and 85 , are used to calculate the nonlinear local acceleration terms in eq. 33 and 34 . It should be noted that $S_{1}$ and $R_{2}$ include velocity terms that are derived from the nonlinear local acceleration terms. This inclusion causes a resonance, as derived in the following.

Because eq 74 and 75 contain $\bar{\epsilon}$, eq 85 must be considered. By substituting eq 72 and 73 into eq 35, the following is obtained: 


$$
A_{3} \frac{\partial v}{\partial x}+B_{3} \frac{\partial v}{\partial y}+C_{3} v \frac{\partial \theta}{\partial x}+D_{3} v \frac{\partial \theta}{\partial y}=-2 \bar{\epsilon}
$$

where

$$
\begin{aligned}
& A_{3}=\cos (\theta \oplus \delta) \\
& B_{3}=-\sin (\theta \oplus \delta) \\
& C_{3}=-\sin (\theta \oplus \delta)
\end{aligned}
$$

and

$$
\mathrm{D}_{3}=-\cos (\theta \oplus \delta) \text {. }
$$

Combining the conditions of one-valuedness for $\tilde{\sigma}, \theta$, and $v$ with eq 74 , 75 , and 88 , six simultaneous (algebraic) linear equations with six unknowns are obtained, that are the local partial derivatives of $\tilde{\sigma}, \theta$, and $\underline{v}$ with respect to $x$ and $y$. Equation 35 , the condition of continuity, may not be considered in the present analysis because it is already recognized in the preceding analysis that the characteristic direction to which eq 35 is related is the stream line.

Calculating the determinant of coefficients of the six simultaneous (algebraic) linear equations yields

$$
\Delta=\Delta_{\mathbf{v}} \Delta_{\sigma}
$$

where

$$
\Delta_{v}=\left|\begin{array}{cc}
\mathrm{A}_{3} & \mathrm{~B}_{3} \\
\mathrm{dx} & \mathrm{dy}
\end{array}\right| \text { and } \Delta_{\sigma}=\left|\begin{array}{cccc}
\mathrm{P}_{1} & \mathrm{Q}_{1} & \mathrm{R}_{1} & \mathrm{~S}_{1} \\
\mathrm{P}_{2} & \mathrm{Q}_{2} & \mathrm{R}_{2} & \mathrm{~S}_{2} \\
\mathrm{dx} & \mathrm{dy} & 0 & 0 \\
0 & 0 & \mathrm{dx} & \mathrm{dy}
\end{array}\right| \text {. }
$$

From $\Delta_{\mathrm{v}}=0$, the activated stress characteristic direction is obtained, which, in this case, is the direction of the slip line. From $\Delta_{\sigma}=0$,

$$
\frac{d y}{d x}=\frac{-\sin 2 \theta\left(1+v^{2} \sin \rho\right) \mp \cos \rho \sqrt{1-v^{4}}}{\cos 2 \theta\left(1+v^{2} \sin \rho\right)+\left(v^{2}+\sin \rho\right)} \text {. }
$$

This transforms to

$$
d y=-\tan (\theta \pm \bar{\delta}) d x
$$

by placing

$$
\begin{aligned}
& 2 \bar{\delta}=\frac{\pi}{2}-\bar{\rho} \quad\left(0 \leqq \rho \leqq \frac{\pi}{2}\right) \\
& \cos \bar{\rho}=\frac{\cos \rho \sqrt{1-v^{4}}}{1+\nu^{2} \sin \rho}
\end{aligned}
$$


and

$$
\sin \rho=\frac{\nu^{2}+\sin \rho}{1+v^{2} \sin \rho}
$$

or, summarizing eq 96,97 , and 98 ,

$$
\tan \bar{\delta}=\sqrt{\frac{1-v^{2}}{1+\nu^{2}}} \tan \delta .
$$

The directions given by eq 95 will be called resonance characteristic directions. These were derived by Geniev (1958) as a particular case of his deformation, but they are still valid, even for general deformation.

When $\mathrm{v}=0$, or when $\nu^{2}=0, \bar{\delta}$ and $\bar{\rho}$ become $\delta$ and $\rho$ respectively, and the resonance characteristic directions coincide with those given by eq 4 . When $v^{2} \leqq 1$, or when $(1 / 2) \gamma \mathrm{v}^{2}<\tilde{\sigma} \sin \rho, \bar{\delta}$ is real, 2 and the resonance characteristic directions are real. However, when $v^{2}>1$, or when $(1 / 2) r v^{2}$ $>\approx \sin \rho, \bar{\delta}$ is imaginary, and the resonance characteristic directions disappear. The only remaining characteristic direction is the stream line. In other words, the system of eq 74, 75, and 85 transfers from the hyperbolic to the quasi-elliptic when $\mathrm{v}$ increases beyond the critical velocity $(2 \tilde{\sigma}$ sin $\rho / \gamma)^{1 / 2}$. This mathematical statement may be restated in physical terms as follows: When $\mathrm{v}$ in the realization of slip lines becomes larger than the critical velocity, the motion of $c-\phi$ material becomes like that of a liquid.

Because the consistency of the system of the fundamental eq $10,11,12$, $27,30,31,32,33,34$ and 35 has been shown, it is now possible to analyze the plastic motion of $c-\phi$ material; however, one difficulty still remains and that is the lack of a general method for determining a. In fact, as may be seen from the following examples, a must be determined in such a manner that the solution exists under given boundary and initial conditions. Therefore, the stress and strain-rate relationship, which is a system of functions of $a$, is also dependent on the boundary and initial conditions. However, the mathematics for this type of problem is not yet known. The following examples are presented with the intention of illustrating the general method for determining a by citing particular cases.

\section{Example 1 - Vibration under the Rankine state}

The velocity field in a ground will be analyzed assuming that the ground surface is horizontal and that all the properties of the ground are functions of depth and time maintaining the same values in the horizontal planes. This is an extension of the Rankine state of stress for inclusion of the velocity field.

For simplicity, only the active state will be considered, where the horizontal plane is the plane of major principal stress. Then, in the lower part of Figure 8, A is the stress point of a horizontal plane under consideration and $B$ is the pole. Moreover, if the right-handed coordinate system is assumed, then

$$
2 \theta=\pi \text {. }
$$

The $+m$ and $-m$ stress characteristic directions are $B D$ and $B E$, where $D$ and $E$ are the $+m$ and $-m$ stress points in contact with the respective limiting lines. Then, as may be recognized from the geometry in Figure 8 , the $+m$ and. $-m$ stress characteristic lines, which are shown in the upper part of Figure 8 as bd and be, form angles of $-\delta$ and $+\delta$ with the vertical, respectively. 


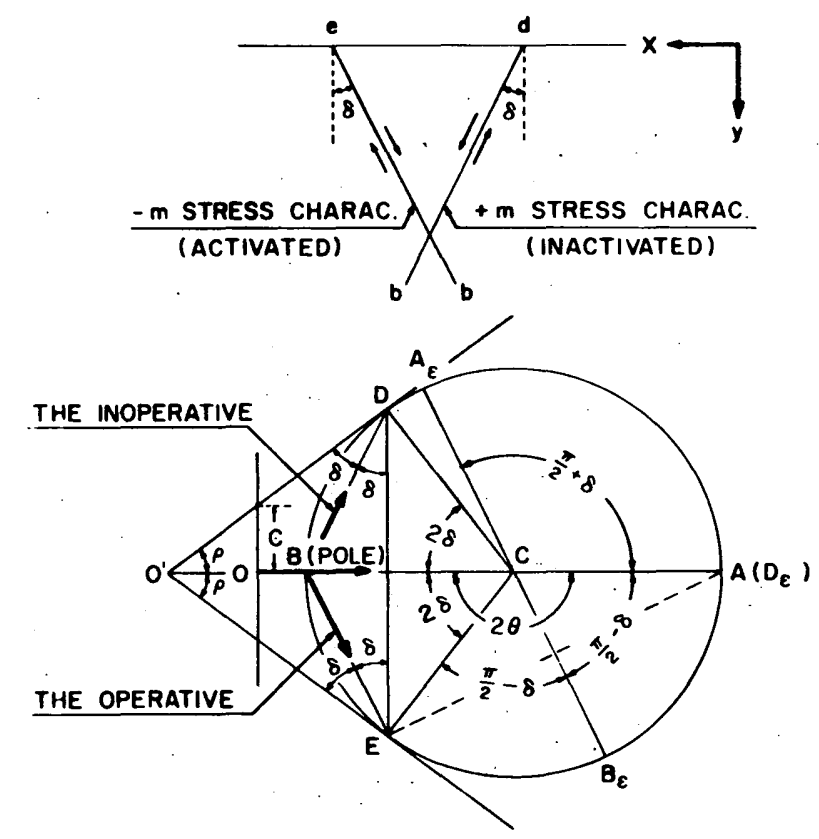

Figure 8. The Rankine state under horizontal ground surface.

Because all the quantities are functions of $y$ and $\underline{t}$,

$$
\dot{\epsilon}_{\mathbf{x}}=0 \text {. }
$$

Equation 101 is equivalent to

$$
\overline{\dot{\epsilon}}=€ \cos 2 a
$$

as may be recognized from the expression for $\dot{\epsilon}$ in eq 10 . Assuming the activation of the $-m$ stress characteristic direction for this case, then

$$
\overline{\dot{\epsilon}}=-\Theta \cos (2 a-2 \delta)
$$

from eq 27. In order to make eq 102 and 103 compatible, $a$ is determined as

$$
2 a=\delta \mp \frac{\pi}{2}
$$

which reduces eq 102 and 103 to the same form

$$
\overline{\dot{\epsilon}}= \pm(\operatorname{\epsilon } \sin \delta \text {. }
$$

The double sign of eq 105, which is not referred to sign $\mathrm{m}$, signifies compression for the upper sign and dilatancy for the lower sign, because () is a positive number and $\bar{\epsilon}$, by definition, is positive when compressive. 
The plastic work rate, $\dot{W}$, for this case, is found to be

$$
\dot{\mathrm{W}}= \pm 2(\cot \rho[\sigma \cos \delta-\mathrm{c} \sin \delta]
$$

in which the double sign is in the same order as in eq 105. Therefore, if $c$ is sufficiently small, dilatancy cannot take place since $W$ must be positive.

Equation 28, the total differential equation along the strain-rate characteristic directions, is for this case

$$
\left(\frac{\partial v}{\partial y} d x+\frac{\partial v}{\partial y} d y\right) d y=0
$$

in which the operative strain-rate characteristic direction is

$$
d y=-\cot \delta d x
$$

as shown from eq 21 , and the inoperative strain-rate characteristic direction is

$$
\mathrm{dy}=0
$$

as shown from eq 29.

By substituting eq 108 into eq 107 , it is found that

$$
\mathrm{v}=\mathrm{v}_{\mathrm{y}} \cos \delta-\mathrm{v}_{\mathrm{x}} \sin \delta
$$

is a function of $t$ only. $\quad V$ is the component of velocity along the operative strain-rate characteristic direction, the positive direction being downward to the right, as may be recognized from the upper part of Figure 8. Since eq 109 satisfies eq 107, another component of velocity is indeterminate in this case, because the equivalence of eq 28 with eq 16 and 27 , as presented at the bottom of p. 10 , does not allow the existence of any other relationship.

The difficulty of determining the component of velocity normal to the operative strain-rate characteristic direction may be avoided by assuming the realization of slip line, in which case

$$
v_{x}=-v(t) \sin \delta
$$

and

$$
v_{y}=v(t) \cos \delta
$$

Reducing $v_{x}$ and $v_{y}$ to functions of $t$ only causes the strain-rate Mohr circle to dwindle to a point, and neither compression nor dilatancy occur in this case.

Equation 109 may be determined from the geometry in Figure 8, in which, for simplicity, the location of $A_{\epsilon}$ is shown for the lower sign of e' 104 only. By the geometric interpretation of eq $26, D_{\epsilon}$ must appear at a point in symmetry with $E$ in respect to $A_{\epsilon} B_{\epsilon}$ and must coincide with $A$, showing that the inoperative strain-rate characteristic direction is horizontal. 
The stress, strain-rate relationships obtained thus far provide equations for determining the distributions of stress and velocity. By substituting the value of $\theta$ given by eq 100 , eq 30,31 , and 32 change to

$$
\begin{aligned}
& \sigma_{x}=\tilde{\sigma} 2 \sin ^{2} \delta-c \cot \rho \\
& \sigma_{y}=\tilde{\sigma} 2 \cos ^{2} \delta-c \cot \rho
\end{aligned}
$$

and

$$
\tau_{\mathrm{xy}}=0
$$

The equations of motion (eq 33 and 34 ) then become

$$
-\frac{d V}{d t} \sin \delta=x
$$

and

$$
\frac{1}{r} \frac{\partial \tilde{\sigma}}{\partial y} 2 \cos ^{2} \delta+\frac{d V}{d t} \cos \delta=Y
$$

on substitution of eq 111 through 115 . The equation of continuity (eq 35) becomes

$$
\frac{\partial \gamma}{\partial t}+V(t) \cos \delta \frac{\partial \gamma}{\partial y}=0
$$

on substitution of eq 111 and 112. Moreover, it is assumed herein that the external force is vibrational:

$$
\mathrm{x}=\mathrm{a} \sin \omega \mathrm{t}
$$

and

$$
\mathrm{Y}=\mathrm{g}
$$

where a and $\omega$ are constants, and $g$ is the acceleration due to gravity.

Then, integration of eq 116 yields

$$
V(t)=\frac{a}{\omega \sin \delta} \cos \omega t+A
$$

where $A$ is a constant. Equation 118 is integrated to yield

$$
y=C \exp \left[B\left(-y \sec \delta+\frac{a}{\omega^{2} \sin \delta} \sin \omega t+A t\right)\right]
$$

in which $B$ and $C$ are constants. Then, eq 117 is integrated to yield

$$
\begin{gathered}
\tilde{\sigma}=-\frac{C}{2 B \cos \delta}(g+a \cot \delta \sin \omega t) \exp [B(-y \sec \delta+ \\
\left.\left.+\frac{a}{\omega^{2} \sin \delta} \sin \omega t+A t\right)\right]+F(t)
\end{gathered}
$$


on the condition that $B$ is not zero, where $F(t)$ is a function dependent on t alone.

$F(t)$ in eq 123 is determined from the boundary condition that $\sigma_{y}=0$ at $y=0$. The constant $A$ may be determined from the initial condition of the plastic motion, if it is known, such as $\mathrm{V}=0$ at $\mathrm{t}=0$. However, deficiencies in the present knowledge of c- $\phi$ material are such that no appropriate method for determining $B$ and $C$ is available.

According to the limitation imposed herein, eq 106 shows the value of the plastic work rate for the $-m$ activation in the active state (the $+m$ or $-m$ activation refers to the activation of the $+m$ or $-m$ stress characteristic direction). It may be shown that eq 106 is also valid for the $+m$ activation in the active state. In the passive state,

$$
\dot{\mathrm{W}}= \pm 2(\dot{\epsilon} \cot \rho[\sigma \sin \delta-\mathrm{c} \cos \delta]
$$

for both the $+m$ and $-m$ activation, in which the upper and lower parts of the double sign correspond to cases of compression and dilatancy, respectively. Therefore, if $c$ is sufficiently small, dilatancy can never occur in any of the cases of the Rankine state.

Before advancing the general theory for practical application, several difficulties must be solved. One of the problems is the theorization of the so-called progressive failure, which is an enlargement of the region of plastic motion or elongation of slip lines, or, in the terms of plasticity of metal, elasto-plastic deformation. It is expected that the progressive failure is related to the particular role of time as a parameter, not as an explicit unknown, in the total differential equations for plastic deformation. The total differential equations are not shown, except for eq 28, because of space limitation. However, since time derivatives are not considered as unknowns in the simultaneous (algebraic) linear equations for deriving characteristic directions, it is clear that any resulting total differential equations do not contain total differentials in regard to time (see Appendix A).

Example 2 - One-dimensional secondary consolidation

The effect of the flow of water on the two-dimensional motion of $c-\phi$ material with the pores filled with water is easily formulated by considering the well-established empirical fact that the intergranular stress alone is responsible for the motion of $c-\phi$ material. Equations 10, 11, 12, 27, 30, 31,32 , and 35 may be used with the convention that $\sigma_{x}, \sigma_{y}$, and $\tau_{x y}$ are the components of intergranular stress, and $\gamma$ is the submerged unit density of $c-\phi$ material. The equations of motion for this case are subject to modification and are obtained by replacing $\sigma_{x}$ and $\sigma_{y}$ in eq 33 and 34 with $\sigma_{x}+p$ and $\sigma_{\dot{y}}+p$, respectively, as follows:

$$
\frac{1}{\gamma}\left(\frac{\partial \sigma_{x}}{\partial x}+\frac{\partial \tau}{\partial y}\right)+v_{x y} \frac{\partial v_{x}}{\partial x}+v_{y} \frac{\partial v_{x}}{\partial y}+\frac{\partial v_{x}}{\partial t}=x-\frac{1}{\gamma} \frac{\partial p}{\partial x}
$$

and

$$
\frac{1}{r}\left(\frac{\partial \tau_{x y}}{\partial x}+\frac{\partial \sigma_{y}}{\partial y}\right)+v_{x} \frac{\partial v_{y}}{\partial x}+v_{y} \frac{\partial v y}{\partial y}+\frac{\partial v_{y}}{\partial t}=Y-\frac{1}{r} \frac{\partial p}{\partial y}
$$

where $\underline{p}$ represents the water pressure. The characteristic directions determined by these equations are the same as those presented previously since, in obtaining the characteristic directions, $\underline{p}$ may be assumed to be a given function of $\underline{x}, \underline{y}$, and $\underline{t}$. 
In order to complete the formulation, the assumption in the theory of three-dimensional consolidation (Biot, 1941; Takagi, 1954) that the rate of volume increase of $c-\phi$ material is equal to the discharge of water out of the volume under consideration will be introduced. Then,

$$
2 \stackrel{\bar{\epsilon}}{\bar{\epsilon}}=\operatorname{div} \mathbf{V}_{\mathbf{w}}
$$

in which $V_{w}$ is the flux of water through a unit area including sections of soil particles and water. $\mathbf{V}_{\mathrm{w}}$ is given by Darcy law as

$$
\mathbf{v}_{\mathbf{w}}=-\mathbf{k} \operatorname{grad} \phi
$$

in which $k$ is the hydraulic coefficient and $\phi$ is the velocity potential. Combining eq 127 and 128 yields

$$
2 \overline{\dot{\epsilon}}=-\operatorname{div}(\mathrm{k} \operatorname{grad} \phi) \text {. }
$$

In eq 129, $\underline{k}$ is a function of $\gamma$ and must be considered as a function of $\underline{x}$, $\underline{y}$, and $\underline{t}$.

Consider a layer of c- $\phi$ material lying on an impermeable bed, extending infinitely in the horizontal direction and filled with water to the surface, that is being consolidated under a uniform load (q per unit area) on the horizontal ground surface. The subject of this analysis is the plastic deformation of the layer, which is known as secondary consolidation. The origin of the $x$ and $y$ axes will be placed on the impermeable bed, the $y$ direction being upward.

The motion of the c- $\phi$ material, in this case, must be vertically downward. Therefore, it is assumed that

$$
\dot{v}_{\mathbf{x}}=0
$$

and that $v_{y}$ is a function of $y$ and $\underline{t}$. Then,

$$
\dot{\epsilon}_{\mathbf{x}}=0
$$

and

$$
\dot{r}_{x y}=0
$$

which are equivalent to

$$
\overrightarrow{\dot{\epsilon}}+\Theta \cos (2 \theta-2 a)=0
$$

and

$$
\text { -(E) } \sin (2 \theta-2 a)=0
$$

respectively, as may be seen from eq 10, 11 , and 12. By making eq 133 and 134 compatible, and considering that compression alone occurs in this case, the following is obtained:

$$
2 \theta-2 a=\pi
$$

and

$$
\overline{\dot{\epsilon}}=Q \text {. }
$$


By substituting eq 136 into eq $27, a$ is determined as

$$
2 a=\pi \mp 2 \delta \text {. }
$$

Then, $\theta$ in eq 135 is determined:

$$
2 \theta=\bigoplus 2 \delta \text {. }
$$

The plastic work rate for this case is

$$
\dot{\mathrm{W}}=2 \Theta \cot \rho[-\mathrm{c}+\Theta \cos \rho] \text {. }
$$

The flow of water causes another kind of energy dissipation - the frictional loss - and the inequality condition for this case is

$$
\dot{w}+k\left(\frac{\partial p}{\partial y}\right)^{2} \geqq 0 .
$$

The stress, strain-rate relationship obtained above is summarized and shown in Figure 9. In accordance with the $+m$ (or the $-m$ ) activation, $A_{+}$(or $A_{-}$) is the major principal strain-rate point, $B_{4}$ (or $B_{-}$) is the minor principal strain-rate point, and the pole $P_{+}$(or $P_{-}$) coincides with $B_{-}$(or $B_{+}$). $P_{+}\left(\right.$or $\left.P_{-}\right)$is shown as the origin of the respective $\dot{\epsilon}, \dot{\gamma}$ axes.

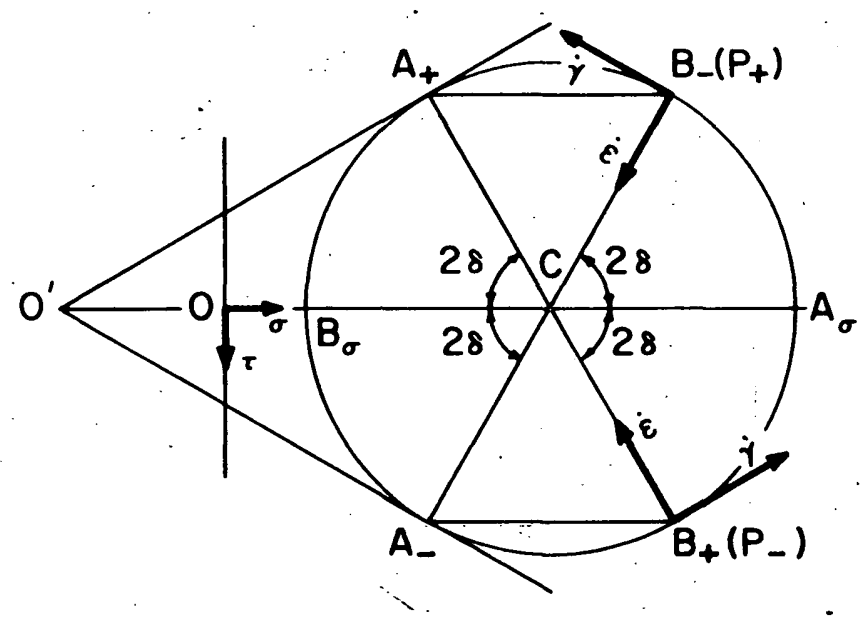

Figure 9. Stress, strain-rate relationship in the one-dimensional secondary consolidation.

Substituting the values of $a$ and $\theta$, thus obtained, into eq 21 and 29, it is found that the operative and the inoperative directions are coincident and horizontal, that is, $d y=0$.. This fact is also illustrated in Figure 9, in which two strain-rate characteristic directions are coincident, $P_{+} A_{+}$(or $P_{-} A_{-}$) in accordance with the $+m($ or $-m)$ activation. Slip lines cannot be developed in this case.

Substituting eq 138 into eq 30,31 , and 32 yields

$$
\begin{aligned}
& \sigma_{x}=\tilde{\sigma}\left(1+\sin ^{2} \rho\right)-c \cot \rho \\
& \sigma_{y}=\tilde{\sigma} \cos ^{2} \rho-c \cot \rho
\end{aligned}
$$


and

$$
\tau_{x y}=\bigoplus \tilde{\sigma} \sin \rho \cos \rho
$$

The state of stress given by eq 141, 142, and 143 is essentially different from the state of stress assumed in the primary consolidation (Takagi, 1954).

For simplicity, it is assumed that no body force is working on the system. Then substituting eq 141,142 , and 143 into eq 125 and 126 yields

$$
\tilde{\sigma}=F(t)
$$

and

$$
\frac{\partial v_{y}}{\partial t}=-\frac{1}{r} \frac{\partial p}{\partial y}
$$

where $F(t)$ is a function of $t$ alone. The nonlinear local partial derivative is neglected in order to obtain eq $145 . F(t)$ will be determined from the boundary condition at the ground surface that

$$
\sigma_{y}+p=q
$$

if $\underline{\mathrm{p}}$ is known on the boundary.

From eq 35 ,

$$
\frac{\partial r}{\partial t}+\frac{\partial\left(r v_{y}\right)}{\partial y}=0 .
$$

Equation,129, under the present assumptions, becomes.

$$
-\frac{\partial v_{y}}{\partial y}=-\frac{\partial}{\partial y}\left(\frac{k}{r_{w}} \frac{\partial p}{\partial y}\right)
$$

which, on integration, yields

$$
v_{y}=\frac{k}{r_{w}} \frac{\partial p}{\partial y}+G(t)
$$

where $G(t)$ is a function of $t$ alone. Using the boundary conditions at the impermeable bed,

$$
v_{y}=0
$$

and

$$
\frac{\partial p}{\partial y}=0
$$

$G(t)$ is found to be equal to zero, and

$$
v_{y}=\frac{k}{r_{w}} \frac{\partial p}{\partial y} .
$$


Combining eq 145 and 152 yields

$$
v_{y}=-\frac{1}{r_{w}} k r \frac{\partial v_{y}}{\partial t} \text {. }
$$

Solution of the simultaneous differential equations (eq 147 and 153) for $y$ and $v_{y}$ will give the solution of this problem. The equations can be solved numerically, provided the functional relationship between $\underline{k}$ and $\gamma$ is given.

It is interesting to note from eq 152 that, in this case, the velocity of $c-\phi$ material, which is directed downward, equals the velocity of water, which is directed upward.

It is well known that the pore pressure in secondary consolidation is almost constant throughout the sample in a consolidometer, but it is the writer's opinion that this empirical fact cannot be used in the analysis. To explain this, the form of water surrounding the particles must be considered. The free water that is not affected by the adsorption force of particles flows first under the pressure of the overburden load. When the free water has disappeared, the outermost part of the adsorbed water will begin to move. Successive initiation of motion of the films of adsorbed water, first the outer films, then those closer to the particle, forms the secondary consolidation. But the pressure that causes the motion of these thin water films, surmounting rather high resistance in the adsorbed water, cannot be measured by a piezometer, which indicates the pressure of stationary water in porous stone.

For rigorous treatment, the immovable part of the adsorbed water must be considered as a part of solid particles, and must be included in $\gamma$. This necessity arises from the simplification of the complicated flow of adsorbed water in the Darcy law. The Darcy law originally takes into account only the flow of free water that is not affected by the adsorption force of particles. If the Darcy law is extended to cover also the flow of adsorbed water, some modifications of the Darcy law such as mentioned above will be necessary.

Because of the difficulties, a complete explanation of secondary consolidation has not yet been attained. However, the validity of the general theory of plastic deformation herein is manifest also in the analysis for Example 2 .

\section{CONCLUSIONS}

A consistent mathematical foundation for plane plastic motion of $c-\phi$ material is presented herein. However, several problems have yet to be solved.

First, the condition for the existence and uniqueness of the solution under given boundary and initial conditions must be investigated using the proper interpretation of the plastic work rate. It is expected that this condition will provide the general method for determining a.

Second, the theory of three-dimensional plastic motion must be developed in order that the assumption adopted in the theory may be compared with empirical results. The most essential requisite for this development will be the geometric interpretation of the three-dimensional tensor, with which the strain-rate tensor and stress tensor must be adequately connected in the same manner as was accomplished herein for the plane plastic motion. 
The geometry suitable for this objective will be geometry on a spherical surface or non-Euclidean elliptic geometry on a plane with suitable geometric interpretation of tensor invariants because these are extensions of the geometry of the Mohr circle used herein.

Third, a theory of pre-plastic deformation, such as that presented by K. Hoshino (1954, 1957, 1961), must be connected with this theory in order that the transfer from the pre-plastic to the plastic deformation may be considered.

Fourth, the three-dimensional yield criterion of $c-\phi$ material is not known a priori, but may be assumed to be represented by eq 7, as was suggested by Drucker and Prager, in accordance with the general theory of plasticity (Appendix D). It should be noted that the three-dimensional yield criterion, if established in this form, gives, on limitation of the mode of motion, various specifications of the yield criterion.

An attempt must be made to solve these problems by enlarging the properties of $c-\phi$ material to make it an idealization of actual soil. Moreover, if engineers are successful in combining irreversible thermodynamics with the three-dimensional theory thus developed, then not only soil mechanics but also snow and ice mechanics may come under their command.

\section{LITERATURE CITED}

Biot, M. A. (1941) General theory of three-dimensional consolidation, Journal of Applied Physics, vol. 12, p. 155.

de Jong, G. de Josselin (1958) The undefiniteness in kinematics for friction material, Brussels Conference on Earth Pressure Problems, Belgian Group of International Society, S. M. F. E., vol. I, p. 56-70.

Drucker, D. C. (1953) Limit analysis of two and three dimensional soil mechanics problems, Journal of the Mechanics and Physics of Solids, vol. 1, p. 217-226.

and Prager, W. (1952) Soil mechanics and plastic analysis or limit design, Quarterly of Applied Mathematics, vol. 2, p. 157-165.

Geniev, G. A. (1958) Voprosy dinamiki sypuchei sredy (Problems of dynamics of pulverulent material), Moscow: Akademiia Stroitel' stva i Arkhitektury SSSR.

Green, A. E. and Zerna, W. (1954) Theoretical elasticity, New York: Oxford University Press.

Hansen, Brinch (1953) Earth pressure calculation, Copenhagen: The Institutions of Danish Civil Engineers.

Hill, R. (1956) The mathematical theory of plasticity. New York: Oxford University Press, p. 16 and 38 .

Hoshino, K. (1954) Tsuchi no rikigaku ni okeru sosei no kihon-riron to sanjiku-shiken e no tekiyo (A basic theory of plasticity in soil mechanics and its application to triaxial test results), Transactions, Japanese Society of Civil Engineers, no. 21 (toxt in Japanese).

(1957) A general theory of mechanics of soils, Proceedings, 4th International Conference, Soil Mechanics and Foundation Engineering, London, vol. 1, p. 160-166. 
Hoshino, K. (1961) An analysis of the volume change, distortional deformation and induced pore pressure of soils under triaxial loading, 5th International Conference, Soil Mechanics and Foundation Engineering, Paris.

Shield, R. T. (1953) Mixed boundary value problems in soil mechanics, Quarterly of Applied Mathematics, vol. 11 p. 61-75.

Sokolovski, V.'V. (1960) Statics of soil media, London: Butterworth.

Takagi, Shunsuke (1954) Atsumitsu gensho no butsuri (Physics of consolidation), Report of the Institute of Science and Technology, University of Tokyo, vol. 8, p. 221-234.

(1958-1960) Doshitsu-rikigaku no kangaekata (Lectures on theories in soil mechanics, Journal, Agricultural Engineering Society of Japan (Tokyo). Part I: vol. 26, no. 4, p. 58-63; Part II: vol. 26, no. 5, p. 41-45; Part III: vol. 26, no. 6, p. 50-55; Part IV: vol. 27, no. 1, p. 44-49; Part V: vol. 27, no. 2, p. 49-54; Part VI: vol. 27, no. 3, p. 38-44; Part VII: vol. 27, no. 4, p. 43-50; Part VIII: vol. 27, no. 6, p. 37-44 (text in Japanese).

Terzaghi, Karl (1951) Theoretical soil mechanics. New York: John Wiley and Sons, Inc., p. 16.

Yamaguchi, H. (1959) Sosei-ryudo ni okeru sokudo-ba no riron (A theory on the velocity field in the plastic flow of granular materials), Transactions, Japanese Society of Civil Engineers, no. 63, p. 8-16 (text in Japanese).

Yoshimura, Y. (1959a) Meta-theory of mechanics of continua subject to deformations of arbitrary magnitudes (Duality of definitions of strain, strain increment and stress for elastic and plastic finite deformations), Aeronautical Research Institute, University of Tokyo, Report no. 343.

(1959b) Sosei-rikigaku (Theory of plasticity). Tokyo: Kyoritsu Publishing Co. (text in Japanese). 
Consider, for simplicity, a set of only two partial differential equations for only two unknown functions $f(x, y)$ and $g(x, y)$ :

$$
a_{1} \frac{\partial f}{\partial x}+b_{1} \frac{\partial f}{\partial y}+c_{1} \frac{\partial g}{\partial x}+d_{1} \frac{\partial g}{\partial y}=e_{1}
$$

and

$$
a_{2} \frac{\partial f}{\partial x}+b_{2} \frac{\partial f}{\partial y}+c_{2} \frac{\partial g}{\partial x}+d_{2} \frac{\partial g}{\partial y}=e_{2}
$$

where $a_{1}$ through $e_{1}$ and $a_{2}$ through $e_{2}$ are given functions of $x, y$, and, in some eases, other parameters. In addition to eq 154 and 155 , the conditions of one-valuedness for $\underline{f}$ and $\underline{g}$ must be taken into account:

$$
\mathrm{df}=\frac{\partial f}{\partial x} d x+\frac{\partial f}{\partial y} d y
$$

and

$$
d g=\frac{\partial g}{\partial x} d x+\frac{\partial g}{\partial y} d y \cdot
$$

Equations 154 through 157 may be considered to form four simultaneous (algebraic) linear equations to determine four unknowns, that is, four partial derivatives of $f$ and $g$ with respect to $x$ and $y$. By placing the determinant, $\Delta$, of the simultaneous equations equal to zero, the characterfistic directions of the partial differential equations, eq 154 and 155, are determined.

When $d x: d y$ represents one of the characteristic directions, the following simultaneous linear equations are solvable, determining the ratio $\lambda: \mu: p: q$.

$$
\begin{aligned}
& \lambda a_{1}+\mu a_{2}=p d x \\
& \lambda b_{1}+\mu b_{2}=p d y \\
& \lambda c_{1}+\mu c_{2}=q d x
\end{aligned}
$$

and

$$
\lambda d_{1}+\mu d_{2}=q d y \cdot
$$

The existence of the solution is assured since $\Delta$ is zero along the characteristic direction. Equations 158 through 161 are constructed by multiplying the rows of $\Delta$ with $\lambda, \mu, p$, and $q$ and adding the results.

Multiply eq 154 and 155 by $\lambda$ and $\mu$, respectively, and add the two equations thus obtained. Then

$$
p d f+q d g=\lambda e_{1}+\mu e_{2} \text {. }
$$

Equation 162 is the total differential equation along the given characteristic direction. This proves Yamaguchi's principle (p. 6). 
This method of finding a total differential equation will be conveniently used to determine the total differential equations along the characteristic directions for the general plastic deformation of $c-\phi$ material. 
The angle of intersection of a pair of conjugate strain-rate characteristic lines is $2 a$ (f) $2 \delta$ (Fig. 7 with substitution of $2 a$ for $\phi$ ) and varies with location and time. The analysis of strain-rate tensor under this variable angle of intersection becomes considerably easier and more systematic with the formulation of invariability of tensors and vectors under coordinate transformation. In this section, the notation of tensor analysis will be used for systematic treatment.

Unit vectors are designated at an arbitrary point under consideration in the operative and the inoperative strain-rate characteristic directions, respectively, by $\mathbf{e}_{0}$ and $\mathbf{e}_{1}$, and those in the $\underline{x}$ and $\underline{y}$ directions, respectively, by $\mathbf{e}_{\mathrm{x}}$ and $\mathbf{e}_{\mathrm{y}}$. Then,

$$
\mathbf{e}_{0}=\mathbf{e}_{\mathbf{x}} \cos (\theta \oplus \delta)-\mathbf{e}_{\mathrm{y}} \sin (\theta \oplus \delta)
$$

and

$$
\mathbf{e}_{1}=\mathbf{e}_{\mathbf{x}} \cos (\theta \mp \delta-2 a)-\mathbf{e}_{\mathrm{y}} \sin (\theta \mp \delta-2 a)
$$

where $\mathbf{e}_{0}$ and $\mathbf{e}_{1}$ are covariant basic vectors. Introduce, in addition to $\mathbf{e}_{0}$ and $\mathbf{e}_{1}$, the contravariant basic vectors, $\mathbf{e}^{0}$
and $\mathbf{e}^{1}$, which are defined by

$$
\mathbf{e}_{\mathbf{j}} \cdot \mathbf{e}^{\mathrm{i}}=\delta_{\mathbf{j}}{ }^{\mathrm{i}}
$$

where $\underline{i}$ and $\underline{j}$ are indexes expressing 0 or 1 , and $\delta_{j}^{i}$ is a Kronecker delta. The dot indicates a scalar product. $\mathbf{e}^{0}$ and $\mathbf{e}^{l}$ are at right angles with $\mathbf{e}_{1}$ and $\mathbf{e}_{0}$, respectively, but are not unit vectors. The vectors $\mathbf{e}^{0}$. and $\mathbf{e}^{l}$ are expressed easily in terms of $\mathbf{e}_{\mathrm{x}}$ and $\mathbf{e}_{\mathrm{y}}$ as follows:

$$
\mathbf{e}^{0} \sin (2 a \oplus 2 \delta)=-\mathbf{e}_{x} \sin (\theta \mp \delta-2 a)-\mathbf{e}_{y} \cos (\theta \mp \delta-2 a)
$$

and

$$
\mathbf{e}^{l} \sin (2 a \oplus 2 \delta)=\mathbf{e}_{\mathrm{x}} \sin (\theta \oplus \delta)+\mathbf{e}_{\mathrm{y}} \cos (\theta \oplus \delta) .
$$

Or simultaneously solving eq 166 and 167 for $\mathbf{e}_{\mathbf{x}}$ and $\mathbf{e}_{\mathrm{y}}$ :

$$
\mathbf{e}_{\mathrm{x}}=\mathbf{e}^{0} \cos (\theta \oplus \delta)+\mathbf{e}^{1} \cos (\theta \subsetneq \delta-2 a)
$$

and

$$
\mathbf{e}_{\mathrm{y}}=-\mathrm{e}^{0} \cos (\theta \oplus \delta)-\mathrm{e}^{1} \sin (\theta \oplus \delta-2 \mathrm{a})
$$

Quantities such as $T_{i j} e^{i} e^{j}, T_{i}^{j} e^{i} e_{j}, T^{i}{ }_{j} e_{i} e^{j}$, and $T^{i j} e_{i} e_{j}$ are called tensors of the second degree, provided they are invariant under coordinate transformation. It is customary to write a vector as $\mathrm{V}^{\mathrm{i}} \mathbf{e}_{\mathrm{i}}$ or $\mathrm{V}_{\mathrm{i}} \mathrm{e}^{\mathrm{i}}$ combining 
components with basic vectors. But this is not done for a tensor. The writer is indebted to $\mathrm{Y}$. Yoshimura $(1959 \mathrm{a}, \mathrm{b})$ for this manner of expressing tensors. In the preceding equations $e^{i} e^{j}$, and so forth, do not signify any kind of product, but merely indicate sequences of two basic vectors. It should be noted that the convention of the dummy index in the tensor analysis is observed. Therefore, $T_{i j} e^{i} e^{j}$, for example, is the sum of four terms. A tensor of the second degree may be expressed by any one of the above four forms. When expressed in the first or the fourth form, a set of four terms, $T_{i j}$ or $T^{i j}$, respectively, is called a set of covariant or contravariant components of a tensor of the second degree. The components of strain-rate tensor with physical meaning must be covariant, because other forms cannot give the well-established law of transformation.

Designating the indexes expressing $\mathrm{x}$ or $\mathrm{y}$ by $\lambda$ and $\mu$, the invariability of the strain-rate tensor under coordinate transformation is expressed as

$$
\dot{\epsilon}_{i j} \mathbf{e}^{i} \mathbf{e}^{j}=\dot{\epsilon}_{\lambda \mu} \dot{e}^{\lambda} \mathbf{e}^{\mu}
$$

It should be noted that $e^{\lambda}$ equals $e_{\lambda}$ in the $\underline{x}, y$ coordinate system, in which the $\underline{x}$ and $y$ directions are fixed and are orthogonal. Substitute eq 168 and 169 into eq 1700, ard čŏmpare the coefficients of $\mathbf{e}^{0} \mathbf{e}^{0}, \mathbf{e}^{0} \mathbf{e}^{1}, \mathbf{e}^{1} \mathbf{e}^{0}$, and. $\mathbf{e}^{1} \mathbf{e}^{1}$. Then,

$$
\begin{gathered}
\dot{\epsilon}_{00}=\dot{\epsilon}_{x x} \cos ^{2}(\theta \oplus \delta)-\dot{\epsilon}_{x y} \sin (2 \theta \oplus 2 \delta) \\
\quad+\dot{\epsilon}_{y y} \sin ^{2}(\theta \oplus \delta) \\
\dot{\epsilon}_{01}=\dot{\epsilon}_{10}=\dot{\epsilon}_{x x} \cos (\theta \oplus \delta) \cos (\theta \bigoplus \delta-2 a)-\dot{\epsilon}_{x y} \sin (2 \theta-2 a) \\
+\dot{\epsilon}_{y y} \sin (\theta \oplus \delta) \sin (\theta \bigoplus \delta-2 a)
\end{gathered}
$$

and

$$
\begin{aligned}
\dot{\epsilon}_{11}=\dot{\epsilon}_{x x} \cos ^{2}(\theta \bigoplus \delta-2 a)-\dot{\epsilon}_{x y} \sin (2 \theta \mp 2 \delta-4 a) \\
+\dot{\epsilon}_{y y} \sin ^{2}(\theta \bigoplus \delta-2 a)
\end{aligned}
$$

where the property

$$
\dot{\epsilon}_{\mathrm{xy}}=\dot{\epsilon}_{\mathrm{yx}}
$$

is used.

Reverting to the original notation, and substituting eq 10,11 , and 12 into eq 171,172 , and 173 yields

$$
\begin{aligned}
& \dot{\epsilon}_{0}=\overline{\dot{\epsilon}}+(\Theta \cos (2 a \oplus 2 \delta) \\
& \dot{\epsilon}_{1}=\overline{\dot{\epsilon}}+(\Theta \cos (2 a \oplus 2 \delta)
\end{aligned}
$$


and

$$
\dot{r}_{01}=\overline{\dot{\epsilon}} \cos (2 a \oplus 2 \delta)+()
$$

When the compression characteristic, eq 27, is used, eq 175, 176, and 177 reduce to

$$
\begin{aligned}
& \dot{\boldsymbol{\epsilon}}_{0}=0 \\
& \dot{\boldsymbol{\epsilon}}_{1}=0
\end{aligned}
$$

and

$$
\dot{r}_{01}=€ \sin ^{2}(2 a \oplus 2 \delta) \text {. }
$$

The following equation defines $\stackrel{\bar{\epsilon}}{\mathrm{E}}$

$$
2 \stackrel{\vec{\epsilon}}{\mathbf{\epsilon}}=\mathrm{g}^{\mathrm{ij}} \dot{\epsilon}_{\mathbf{i j}}
$$

in which $\mathrm{g}^{\mathrm{ij}}$ is given (Yoshimura, 1959a, b; Green and Zerna, 1954) by $e^{i} \cdot e^{j}$. Equation 181 becomes the compression characteristic when eq 166 , $167,175,176$, and 177 are substituted in the right-hand side.

The tensor analysis introduced in this manner in Euclidean geometry is much simpler and more comprehensible than the tensor analysis in Riemannian geometry, in which the concept of basic vectors cannot be used consistently. The reason for this is that the Riemannian geometry is constructed as a specification of the Euclidean geometry by omitting an appropriately chosen number of pairs of basic vectors in the latter. As long as algebraic calculation alone is concerned, the basic vectors in both geometries are used consistently. When, however, differentiation of vectors and tensors is introduced, the use of basic vectors must be abandoned in the case of Riemannian geometry because the derivative of a vector in the Riemannian space, which is a part of the Euclidean space, cannot necessarily be confined in the original Riemannian space. In order to obtain a clear description of the Euclidean geometry, it is advisable to use the combination of components and basic vectors instead of using components alone, as was done in the Riemannian geometry. For example, it will be found that all the formulas for rectangular coordinates are valid intact even for the curvilinear coordinates, if combinations of components and basic vectors are adopted to express vectors and tensors. 
Consider $(\sigma)$ and $\bar{\sigma}$ to be the radius and the $\sigma$ coordinate of the center, respectively, of the Mohr circle of stress, as shown in Figure 3 . Then eq 30,31 , and 32 may be written in another form:

$$
\begin{aligned}
& \sigma_{x}=\bar{\sigma}+\sigma \cos 2 \theta \\
& \sigma_{y}=\bar{\sigma}-\sigma \cos 2 \theta
\end{aligned}
$$

and

$$
\tau_{\mathrm{xy}}=-\sigma \sin 2 \theta
$$

where

$$
\text { ( })=\tilde{\sigma} \sin \rho=\bar{\sigma} \sin \rho+c \cos \rho .
$$

In order to obtain the stress, strain-rate relationship in harmonious form, eq 182,183 , and 184, rather than eq 30,31 , and 32 , must be used with eq 10,11 , and 12 and eq 27 to eliminate $\theta$, because $\sigma$ and $\bar{\sigma}$ are the same type of tensor invariants as (E) and $\overline{\dot{\epsilon}}$.

The following is one of the expressions of the stress, strain-rate relationship:

$$
\begin{aligned}
& \text { (ङ) } \dot{\epsilon}_{x}=\frac{\sigma_{x}}{2} \cos \rho \cos (\rho \oplus 2 a)-\frac{\sigma_{y}}{2}[\cos 2 a+\sin \rho \sin (\rho \oplus 2 a)]- \\
& -\tau_{x y} \sin 2 a-c \cos \rho \sin (\rho \mp 2 a) \\
& \frac{\sigma}{(\epsilon)} \dot{\epsilon}_{y}=\frac{\sigma y}{2} \cos \rho \cos (\rho \bigoplus 2 a)-\frac{\sigma x}{2} \cdot[\cos 2 a+\sin \rho \sin (\rho \mp 2 a)]+ \\
& +\tau_{x y} \sin 2 a-c \cos \rho \sin (\rho \bigoplus 2 a) \\
& \frac{\text { (ब) }}{\epsilon} \dot{\gamma}_{\mathrm{xy}}=\tau_{\mathrm{xy}} \cos 2 a+\frac{\sigma_{\mathrm{x}}-\sigma_{\mathrm{y}}}{2} \sin 2 a \text {. }
\end{aligned}
$$

However, the most charming of the several possible expressions of the stress, strain-rate relationship may be the following matrix representation with reduction of the tensors to deviatoric:

$$
\frac{1}{(\epsilon)}\left[\begin{array}{ll}
\dot{\epsilon}_{\mathrm{x}}^{\prime} & \dot{\gamma}_{\mathrm{xy}} \\
\dot{\gamma}_{\mathrm{xy}} & \dot{\epsilon}_{\mathrm{y}}^{\prime}
\end{array}\right]=\frac{1}{\sigma}\left[\begin{array}{cc}
\cos 2 a & -\sin 2 a \\
\sin 2 a & \cos 2 a
\end{array}\right]\left[\begin{array}{cc}
\sigma_{\mathrm{x}}^{\prime} & \tau_{\mathrm{xy}} \\
\tau_{\mathrm{xy}} & \sigma_{\mathrm{y}}^{\prime}
\end{array}\right]
$$

where a prime signifies that the respective quantity is deviatoric. Equation 189 shows that the two deviatoric tensors are connected by a matrix, as if a $c-\phi$ material is an anisotropic material.

When $a=0$, the following relationships exist:

$$
\frac{1}{2(\epsilon)} \dot{\epsilon}_{x}=\frac{\partial}{\partial \sigma_{x}}(\sigma-\bar{\sigma} \sin \rho)
$$


36

$$
\begin{aligned}
& \frac{1}{2(\epsilon)} \dot{\epsilon}_{\mathrm{y}}=\frac{\partial}{\partial \sigma_{\mathrm{y}}}(\sigma-\bar{\sigma} \sin \rho) \\
& \frac{1}{\xi} \dot{r}_{\mathrm{xy}}=\frac{\partial}{\partial \tau_{\mathrm{xy}}}(\sigma-\bar{\sigma} \sin \rho)
\end{aligned}
$$

where

$$
\sigma^{2}=\left(\frac{\sigma_{x}-\sigma_{y}}{2}\right)^{2}+\tau_{x y}^{2}
$$

and

$$
\bar{\sigma}=\frac{\sigma_{\mathbf{x}}+\sigma_{\mathrm{y}}}{2}
$$

Therefore,

$$
\psi=\sigma-\bar{\sigma} \sin \rho
$$

is a plastic potential in plane deformation for the case in which Saint-Venant's postulate is valid. It should be noticed that

$$
\psi=c \cos \rho
$$

is the Mohr criterion of yielding. The validity of the concept of plastic potential in the Drucker and Prager plane deformation of $c-\phi$ material is manifest in this analysis. 
By placing $a=0$ in eq 186,187 , and 188 , the following is obtained:

$$
\begin{aligned}
& \frac{\sigma}{\epsilon} \dot{\epsilon}_{x}=\frac{\cos ^{2} \rho}{2} \sigma_{x}-\frac{1+\sin ^{2} \rho}{2} \sigma_{y}-c \sin \rho \cos \rho \\
& \frac{\sigma}{\epsilon} \dot{\epsilon}_{y}=\frac{\cos ^{2} \rho}{2} \sigma_{y}-\frac{1+\sin ^{2} \rho}{2} \sigma_{y}-c \sin \rho \cos \rho
\end{aligned}
$$

and

$$
\frac{\partial}{\epsilon} \dot{r}_{x y}=\tau_{x y} \text {. }
$$

When $x$ and $y$ are the principal axes, eq 197, 198, and 199 may be abbreviated as follows:

$$
\overbrace{\epsilon} \dot{\epsilon}_{1}=\frac{\cos ^{2} \rho}{2} \sigma_{i}-\frac{1+\sin ^{2} \rho}{2} \sigma_{j}-c \sin \rho \cos \rho
$$

in which a set of indexes, $i$ and $j$, represents the two sets of cyclic permutations of the indexes 1 and $\bar{\Sigma}$ of the principal axes.

The generalization of eq 200 to three-dimensional deformation may be:

$$
\mathrm{K} \dot{\epsilon}_{\mathrm{p}}=\dot{\sigma}_{\mathrm{p}}-v\left(\sigma_{\mathrm{q}}+\sigma_{\mathrm{r}}\right)-\mathrm{A}
$$

where $\mathrm{K}, \nu$, and $\mathrm{A}$ are undetermined constants, and the indexes $\mathrm{p}, \mathrm{q}$, and $\underline{\mathrm{r}}$ represent the three sets of cyclic permutations of the indexes 1,2 , and 3 of the principal axes.

In the case of the plane strain, it may be assumed that

$$
\dot{\boldsymbol{\epsilon}}_{3}=0
$$

and

$$
\sigma_{3}=v\left(\sigma_{1}+\sigma_{2}\right)+A \text {. }
$$

Then,

$$
\mathrm{K} \dot{\epsilon}_{\mathrm{i}}=\sigma_{\mathrm{i}}\left(1-v^{2}\right)-\sigma_{\mathrm{j}}\left(v+v^{2}\right)-\mathrm{A}(1+v) \text {. }
$$

Comparing eq 204 with eq 200 , it is found that

$$
\leqslant \quad v=\frac{1+\sin ^{2} \rho}{2}
$$

and

$$
A=c \sin \rho \cos \rho \text {. }
$$

Determined in this manner, $K$ is referred to as the two-dimensional tensor invariant, and cannot be used in the three-dimensional deformation. 
A three-dimensional plastic potential $\psi_{3}$ may be defined by

$$
\frac{\partial \stackrel{⿹ 勹}{\Psi}_{3}}{\partial \sigma_{\lambda}}=K \dot{\epsilon}_{\lambda} \text {. }
$$

with $\lambda=1,2$, and 3 . Substituting eq 201 into the right-hand side of eq 207 and integrating the three equations thus obtained yields

$$
\begin{gathered}
\psi_{3}=\frac{1}{2}\left(\sigma_{1}^{2}+\sigma_{2}^{2}+\sigma_{3}^{2}\right)-v\left(\sigma_{1} \sigma_{2}+\sigma_{2} \sigma_{3}+\sigma_{3} \sigma_{1}\right) \\
-\mathrm{A}\left(\sigma_{1}+\sigma_{2}+\sigma_{3}\right)+\mathrm{B}
\end{gathered}
$$

in which $B$ is an integration constant, and $v$ and $A$ are given by eq 205 and 206. By using the relationships

$$
\sigma_{1}^{2}+\sigma_{2}^{2}+\sigma_{3}^{2}=\frac{1}{3} \mathrm{~J}_{1}^{2}+2 \mathrm{~J}_{2}
$$

and

$$
\sigma_{1} \sigma_{2}+\sigma_{2} \sigma_{3}+\sigma_{3} \sigma_{1}=\frac{1}{3} \mathrm{~J}_{1}^{2}-\mathrm{J}_{2}
$$

$\psi_{3}$ changes to

$$
\psi_{3}=J_{2}(1+v)-\frac{\sin ^{2} \rho}{6} \cdot J_{1}^{2}-J_{1} c \sin \rho \cos \rho+B
$$

Therefore, by placing

$$
B=-\frac{3}{2} c^{2} \cos ^{2} \rho
$$

it follows that

$$
\psi_{3}=\frac{1}{2}\left(3+\sin ^{2} \rho\right) \quad\left[J_{2}-\lambda^{2}\left(J_{1}+3 \cot \rho\right)^{2}\right]
$$

by introducing $\lambda$ which is defined by

$$
\lambda=\frac{\sin \rho}{\sqrt{3\left(3+\sin ^{3} \rho\right)}} .
$$

It is assumed that $\psi_{3}=0$ is the yield criterion for the three-dimensional plastic deformation of $c-\phi$ material, then, all of the allowable states of stress must be in the region defined by

$$
\psi_{3} \leqq 0
$$

because particular sets of the allowable state of stress $\sigma_{1}=\sigma_{2}=\sigma_{3}$ are contained in this region. Solving the inequality (eq 2.15), it is found that all the allowable stresses are contained either in the region defined by 


$$
\mathrm{J}_{2}^{1 / 2} \leqq \lambda\left(\mathrm{J}_{1}+3 \mathrm{c} \cot \rho\right)
$$

or in the region defined by

$$
\text { c } \quad-\mathrm{J}_{2}{ }^{1 / 2} \geqq \lambda\left(\mathrm{J}_{1}+3 \mathrm{c} \cot \rho\right)
$$

since the boundary of these regions is the yield criterion. By choosing the one region that contains the allowable state of stress $\sigma_{1}=\sigma_{2}=\sigma_{3}=\infty$ it is found that the region of eq 216 is allowable, but the region of eq 217 is not allowable. Therefore, it is seen, as proposed by Drucker and Prager (1953), that

$$
\mathrm{J}_{2}{ }^{1 / 2}=\lambda \mathrm{J}_{1}+\mathrm{k}
$$

where

$$
k=3 \lambda \mathbf{c} \cot \rho
$$

is a yield criterion for three-dimensional deformation, and moreover, it is seen that eq 8 represents a plastic potential.

One of the difficulties in formulating the three-dimensional theory of $c-\phi$ material is that the three-dimensional extension of the Mohr criterion of yielding is not known a priori. However, eq 218 may be assumed to be the three-dimensional yield criterion of $c-\phi$ material because, although SaintVenant's postulate has been assumed, eq 218 has been proven to be the threedimensional extension of the Mohr criterion of yielding. Furthermore, eq 218 is the simplest of any possible expressions.

As shown by Hill (1956, p. 15), the three-dimensional yield criterion of the most general isotropic plastic material is a function of $\mathrm{J}_{1}, \mathrm{~J}_{2}$, and $\mathrm{J}_{3}$ where $\mathrm{J}_{3}$ is the third invariant of stress tensor. Then, if it is assumed that eq 218 is the three-dimensional yield criterion, it must be admitted that $\lambda$ and $\mathrm{k}$ can contain $\mathrm{J}_{3}$. It is evident that the three-dimensional yield criterion thus established will contain the intermediate principal stress $\sigma_{\text {II }}$ in addition to $\sigma_{I}$ and $\sigma_{\text {III }}$

It must also be assumed, in accordance with the general theory of plas ticity, that the effect of work-hardening (the hysteresis effect) is contained in $\lambda$ and $\underline{k}$ in order that the implication of cohesion may be explained. It must be noted that when the mode of motion is limited, the three-dimensional yield criterion in this form gives various specifications of the yield criterion. It seems hopeful to assume this form as the three-dimensional yield criterion of $c-\phi$ material. An attempt must be made to establish the three-dimensional yield criterion in such a form as an idealization of actual soil. 


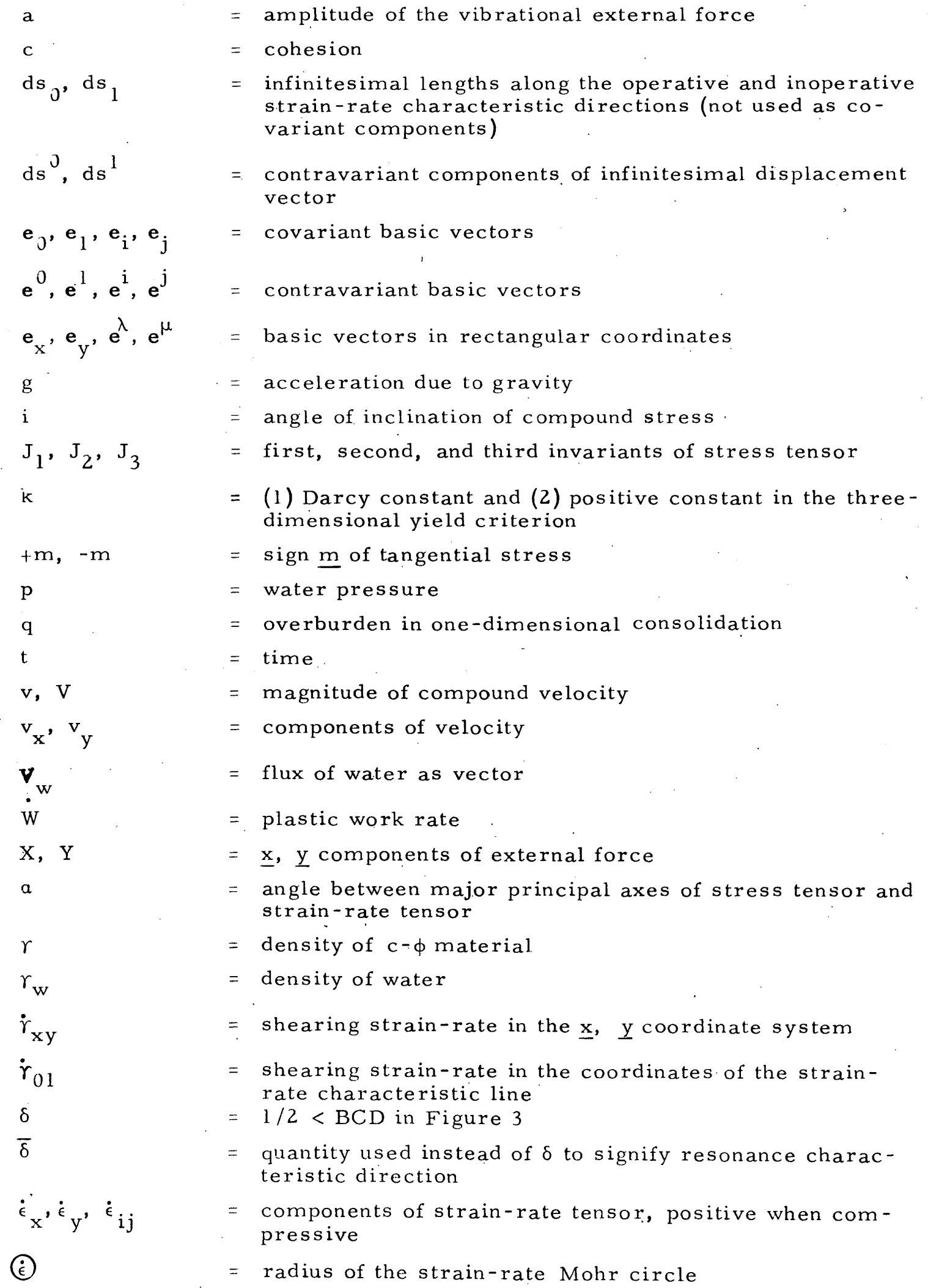


$\tilde{\sigma}$

$\sigma_{I}, \sigma_{\text {III }},{ }^{\sigma I I}$

$\sigma_{1}, \sigma_{2}, \sigma_{3}$

$$
\tau_{\mathrm{xy}}, \tau
$$

$\phi$

$\psi, \psi_{\mathrm{d}}$

$\omega$

(†)

\section{APPENDIX E}

$=\dot{\epsilon}$ coordinate of the center of the strain-rate Mohr circle, one-balf the rate of volume decrease

= angle from the plane of major principal stress to the vertical plane

$=$ positive constant in the three-dimensional yield criterion

$=$ nondimensional velocity signifying resonance characteristic direction

$=$ frictional angle

$=$ quantity used instead of $\rho$ to signify resonance characteristic direction

= components of stress tensor, positive when compressive

$=$ radius of the stress Mohr circle

$=\sigma$ coordinate of the center of the stress Mohr circle

$=\overline{\mathrm{O}^{\prime} \mathrm{C}}$ in Figure 3

$=$ principal stresses arranged in the order of magnitude

$=$ principal stresses arranged in an arbitrary order

$=$ shear stress

$=$ (1) $1 / 2$ of the angle $E_{\sigma} \mathrm{CE}_{\epsilon}$ in Figure 5 or $1 / 2$ of the angle $D_{\sigma} C D_{\epsilon}$ in Figure 6 and (2) the velocity potential of the ground water flow

= plastic potential

$=$ (1) angle from the $\mathbf{x}$ axis to the direction of velocity and (2) the quantity signifying vibrational force

$=$ sign notation used where either the upper or the lower of the double sign should be taken in accordance with the activation of $+m$ or $-m$ stress characteristic direction 


\section{Unclassified}

Security Classification

DOCUMENT CONTROL DATA - R\&D

(Security classification of title, body of abstract and indexing annotation must be entered when the overall report is claseified) 1. ORIGINATIN G ACTIVITY (Comporate author)

U.S. Army Cold Regions Research and Engineering Laboratory, Hanover, N. H. Unclassified

3. REPORT TITLE

\section{PLANE PLASTIC DEFORMATION OF SOILS}

\section{DESCRIPTIVE NOTES (Type of roport and inclusive dates)}

Research Report

5. AUTHOR(S) (Last name, first name, initial)

Takagi, Shunguke

\begin{tabular}{|c|c|c|}
\hline $\begin{array}{r}\text { 6. REPORT DATE } \\
\text { July } 1966\end{array}$ & $\begin{array}{l}\text { 7a. TOTAL NO. OF PAGES } \\
44\end{array}$ & $\begin{array}{c}\text { 7b. NO. OF REFS } \\
19\end{array}$ \\
\hline $\begin{array}{l}\text { Ba. CONTRACT OR GRANT NO. } \\
\text { b. PROJECT NO. }\end{array}$ & $\begin{array}{l}\text { 9a. ORIGINATOR'S REPORT } \\
\text { Research Report }\end{array}$ & $\operatorname{BER}(S)$ \\
\hline $\begin{array}{l}\text { c. DA Task IV014501 B52A02 } \\
\text { d. }\end{array}$ & 9b. OTHER REPORT NO(S) & other numbers that may be assicnod \\
\hline
\end{tabular}

10. AVAILABILITY/LIMITATION NOTICES

Distribution of this document is unlimited

11. SUPPL EMEN TARY NOTES

12. SPONSORING MILITARY ACTIVITY

U.S. Army Cold Regions Research and Engineering Laboratory

13. ABSTRACT

A consistent theory of plane plastic deformation of soil is formulated by assuming soil as an ideal material that has constant cohesion and friction angle. Such an ideal soil is an extension of the ideal metal that has, in the terminology of soil mechanics, cohesion only. After a review of the existing theories from which the present theory has emerged, the mathematical expression referred to as the "compression characteristic" is developed. Then the system of differential equations is shown by the theory of characteristic lines. Many mathematical and physical problems remain to be solved before the perfect explanation of the plasticity of ideal soil will be attained. 


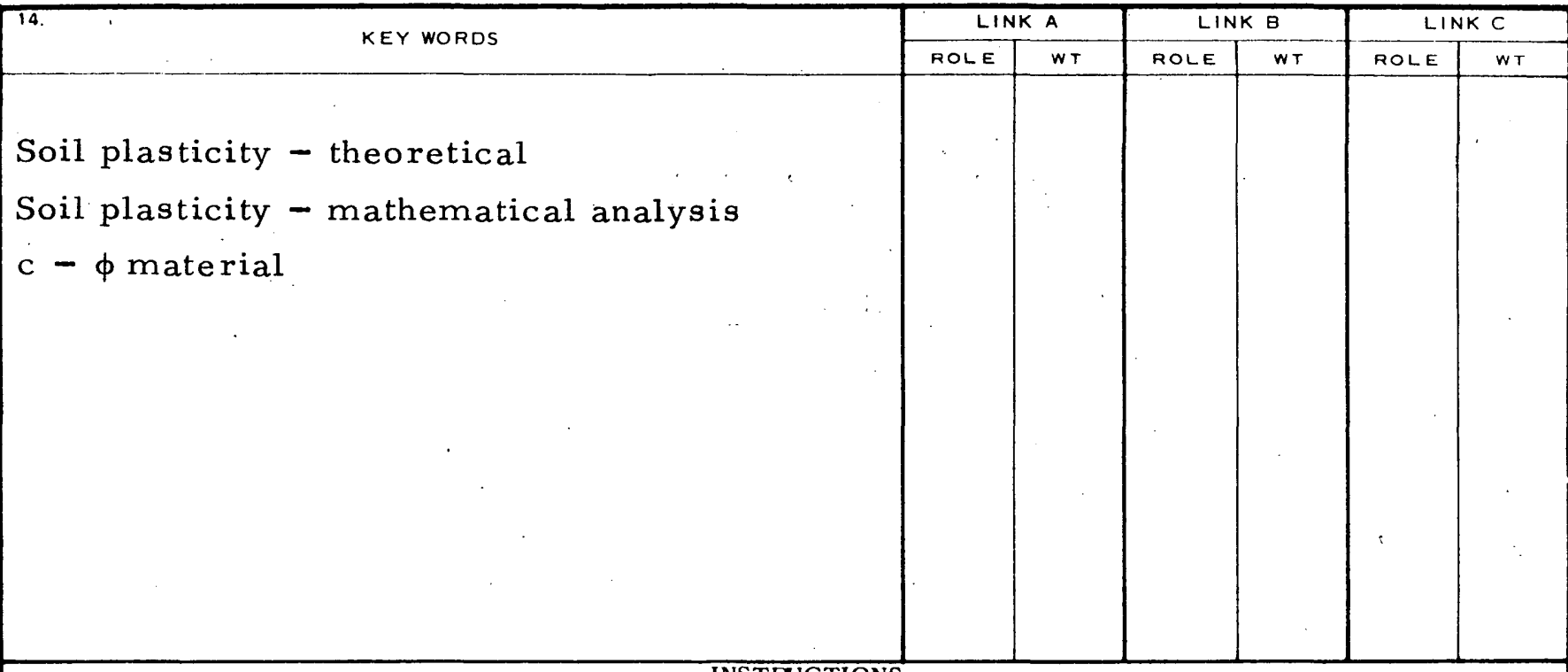

\section{INSTRUCTIONS}

1. ORIGINATING ACTIVITY: Enter the name and address of the contractor, subcontractor, grantee, Department of Defense activity or other organization (corporate author) issuing the report.

2a. REPORT SECURITY CLASSIFICATION: Enter the overall security classification of the report. Indicate whether "Restricted Dat a" is included. Marking is to be in accordance with appropriate security regulations.

2b. GROUP: Automatic downgrading is specified in DoD Directive 5200.10 and Armed Forces Industrial Manual. Enter the group number. Also, when applicable, show that optionat markings have been used for Group 3 and Group 4 as authorized.

3. REPORT TITLE: Enter the complete report title in all capital letters. Titles in all cases should be unclassified. If a meaningful title cannot be selected without classification, show title classification in all capitals in parenthesis immediately following the title.

4. DESCRIPTIVE NOTES: If appropriate, enter the type of report, e.g., interim, progress, summary, annual, or final. Give the inclusive dates when a specific reporting period is covered.

5. AUTHOR(S): Enter the name(s) of author(s) as shown on or in the report. Enter last name, first name, middl e initial. If military, show rank and branch of service. The name of the principal author is an absolute minimum requirement.

6. REPORT DATE: Enter the date of the report as day, month, year; or month, year. If more than one date appears on the report, use date of publication.

7a. TOTAL NUMBER OF PAGES: The total page count should follow normal pagination procedures, i. e., enter the number of pages containing information.

7b. NUMBER OF REFERENCES: Enter the total number of references cited in the report.

8a. CONTRACT OR GRANT NUMBER: If appropriate, enter the applicable number of the contract or grant under which the report was written.

$8 b ; 8 c, 88 d$. PROJECT NUMBER: Enter the appropriate military department identification, such as project number, subproject number, system numbers, task number, etc.

9a. ORIGINATOR'S REPORT NUMBER(S): Enter the official report number by which the document will be identified and controlled by the originating activity. This number must be unique to this report.

9b. OTHER REPORT NUMBER(S): If the report has been assigned any other report numbers (either by the originator or by the sponsor), al so enter this number(s).
10. AVAILABILITY/LIMITATION NOTICES: Enter any limitations on further dissemination of the report, other than those imposed by security classification, using standard statements such as:

(1) "Qualified requesters may obtain copies of this report from DDC."

(2) "Foreign announcement and dissemination of this report by DDC is not authorized."

(3) “U. S. Government agencies may obtain copies of this report directly from DDC. Other qualified DDC users shall request through $\therefore$

(4) "U. S. military agencies may obtain copies of this report directly from DDC. Other qualified users shall request through $\therefore$

(5) "All distribution of this report is controlled. Qualified DDC users shall request through "

If the report has been furnished to the Office of Technical Services, Department of Commerce, for sale to the public, indicate this fact and enter the price, if known.

i1. SUPPLEMENTARY NOTES: Use for additional explanatory notes.

12. SPONSORING MILITARY ACTIVITY: Enter the name of the departmental project of fice or laboratory sponsoring (pay ing for) the research and development. Include address.

13. ABSTRACT: Enter an abstract giving a brief and factual summary of the document indicative of the report, even though it may also appear elsewhere in the body of the technical report. If additional space is required, a continuation sheet shall be attached.

It is highly desirable that the abstract of classified reports be unclassified. Each paragraph of the abstract shall end with an indication of the military security classification of the information in the paragraph, represented as (TS), (S), (C), or $(U)$.

There is no limitation on the length of the abstract. However, the suggested length is from 150 to 225 words.

14. KEY WORDS: Key words are technically meaningful terms or short phrases that characterize a report and may be used as index entries for cataloging the report. Key words must be selected so that no security classification is required. Idenfiers, such as equipment model designation, trade name, military project code name, geopraphic location, may be used as key words but will be followed by an indication of technical context. The assignment of links, rules, and weights is optional. 